\title{
Transport equations and linear response of superfluid Fermi mixtures in neutron stars
}

\author{
Mikhail E. Gusakov \\ Ioffe Physical Technical Institute, Politekhnicheskaya 26, 194021 Saint-Petersburg, Russia
}

\begin{abstract}
We study transport properties of a strongly interacting superfluid mixture of two Fermi-liquids. A typical example of such matter is the neutron-proton liquid in the cores of neutron stars. To describe the mixture, we employ the Landau theory of Fermi-liquids, generalized to allow for the effects of superfluidity. We formulate the kinetic equation and analyze linear response of the system to vector (e.g., electromagnetic) perturbation. In particular, we calculate the transverse and longitudinal polarization functions for both liquid components. We demonstrate, that they can be expressed through the Landau parameters of the mixture and polarization functions of noninteracting matter (when the Landau quasiparticle interaction is neglected). Our results can be used, e.g., for studies of the kinetic coefficients and low-frequency long-wavelength collective modes in superfluid Fermimixtures.
\end{abstract}

PACS numbers:

\section{INTRODUCTION}

Neutron stars are ultracompact objects with radius $R \sim 10 \mathrm{~km}$ and a mass $\sim M_{\odot}$, where $M_{\odot} \approx 1.99 \times 10^{33} \mathrm{~g}$ is the solar mass. The density in the cores of neutron stars is a few times larger than the density $\rho_{0} \approx 2.8 \times 10^{14} \mathrm{~g} \mathrm{~cm}^{-3}$ in atomic nuclei. The simplest matter composition in the internal layers of neutron stars includes neutrons, protons, and electrons. Neutrons and protons form a strongly nonideal, degenerate Fermi-liquid, while electrons can be considered as a degenerate gas of free relativistic particles. It is generally accepted [1 $[3]$ that at low enough temperatures $T \lesssim\left(10^{8}-10^{10}\right) \mathrm{K}$ neutrons and protons become superfluid. Thus, to compare the theory with observations of neutron stars one should be able to describe a strongly nonideal, superfluid mixture of Fermi-liquids. In particular, the kinetic properties of such matter are of primary interest.

One of the important problems in the kinetics of superfluid mixtures is the calculation of the so called response functions that characterize response of the system to small external perturbations. The response functions determine, for example, the emissivity of various neutrino processes (e.g., the Cooper-pairing neutrino emission processes [4 9] or bremsstrahlung processes [10]) and thus influence the thermal evolution of neutron stars. Furthermore, the formalism of response functions is ideally suited to study collective modes in the system which may lead to a discovery of new exotic processes of neutrino emission. Finally, the vector response functions determine the screening properties of particle interaction, influence particle collision amplitudes and hence affect kinetic coefficients.

Although for normal matter of neutron stars the response functions and the collective degrees of freedom have been extensively discussed in the literature (see, e.g., Refs. [11 22]), the author is unaware of any discussion concerning the response in superfluid strongly interacting mixtures. However, for a one-component strongly nonideal superfluid Fermi-liquid (neutrons in the crust of a neutron star), the response functions were calculated recently by Leinson [9]. He generalized the results of Leggett [23] to the case of not too small wave vectors and perturbation frequencies and calculated the response of the system to axial-vector perturbation.

The same problem was also examined in Ref. [6]. The authors of this reference considered a superfluid neutronproton mixture; however, they do not allow for interaction between neutrons and protons. Therefore, de facto they analyze the system of two noninteracting one-component Fermi-liquids. The results obtained in Ref. [6] were discussed (and criticized) in Ref. [9].

It is convenient to calculate the response functions using the method of kinetic equation. For that one has to formulate the kinetic equation which correctly describes (a) possible superfluidity of neutrons and protons and (b) various Fermi-liquid effects connected with interactions between neutrons and protons. For a one-component Fermiliquid such an equation was derived, for example, by Betbeder-Matibet and Nozieres [24] (see also Ref. 25]). Neglecting the Fermi-liquid effects the superfluid kinetic equation was thoroughly examined, e.g., in Refs. 26 30] (see also references therein).

There exist a number of papers exploring the transport properties of superfluid matter in the cores of neutron stars (see, e.g., Refs. [31 35]). However, to our best knowledge, the kinetic equation, satisfying both conditions (a) and (b), has not yet been proposed.

Partially, this is because most studies in the literature have been devoted to kinetics of electron gas, in particular to electron kinetic coefficients. To calculate these coefficients the kinetic equations for nucleons are not required since one usually neglects the interaction between neutrons and electrons and considers protons only as scatterers. Yet another reason is the one-component Fermi-liquid (for example, electrons in metals or liquid helium-3), for which the 
Fermi-liquid effects are well known to play no major role in a variety of kinetic phenomena.

Here we demonstrate that the situation with the superfluid mixtures is different and that the Fermi-liquid effects can be more pronounced there. To a large extent this is due to the mean-field interaction between neutron and proton quasiparticles. In the hydrodynamics of superfluid mixtures 36 41] this interaction leads to entrainment of neutrons by the superfluid motion of protons and vice versa (the so called entrainment effect). We will show that the similar effects are important for the kinetics of superfluid mixtures.

In the present paper we formulate the collisionless kinetic equation, satisfying both conditions (a) and (b). Employing this equation, we calculate and analyze response of the system to a vector (electromagnetic) perturbation (more precisely, we calculate the longitudinal and transverse polarization functions). To describe the superfluid mixture we use the framework of the Landau theory of Fermi-liquids, extended by Larkin and Migdal [42, 43] and by Leggett [23, 44] to allow for superfluidity. For simplicity, we assume that in thermodynamic equilibrium both particle species pair in the spin-singlet ${ }^{1} \mathrm{~S}_{0}$ state (this is indeed a simplification since, according to microscopic calculations [2], neutrons in the cores of neutron stars pair in the spin-triplet ${ }^{3} \mathrm{P}_{2}$ state).

In the collisionless approximation, which we are interested in here, the electrons interact with the nucleons only through a self-consistent electromagnetic field. Thus, in what follows, the electrons can be safely ignored; this does not change our results.

The paper is organized as follows. In Sec. II we derive the kinetic equation describing, in the linear approximation, mixtures of superfluid Fermi-liquids. In Sec. III we calculate and analyze the longitudinal and transverse polarization functions for mixtures. In Sec. IV we propose a nonlinear kinetic equation describing superfluid mixtures in the quasiclassical limit. Section V presents the summary.

Below, unless otherwise stated, we use the system of units in which the Planck constant $\hbar$, the speed of light $c$, the Boltzmann constant $k_{\mathrm{B}}$, and the normalization volume $V$ equal unity, $\hbar=c=k_{\mathrm{B}}=V=1$.

\section{THE KINETIC EQUATION FOR SUPERFLUID FERMI MIXTURES}

\section{A. Thermodynamic equilibrium}

To establish notations, let us briefly consider a strongly interacting degenerate homogeneous Fermi-liquid in thermodynamic equilibrium at some temperature $T$. We assume that the liquid is two-component and composed of particles of two species, $i=1$ and $i=2$. Here and below the indices $i$ and $j$ refer to these species.

Weakly excited states of our system can be described in terms of quasiparticles. According to the Landau theory of Fermi-liquids, in the normal (nonsuperfluid) matter, the energy $\varepsilon_{\boldsymbol{k}}^{(i)}$ of a quasiparticle $i$ with momentum $\boldsymbol{k}$ is a functional of quasiparticle distribution functions $\mathcal{N}_{\boldsymbol{k}}^{(j=1,2)}$ (see, e.g., Refs. [45, 446]),

$$
\varepsilon_{\boldsymbol{k}}^{(i)}\left[\mathcal{N}_{\boldsymbol{k}}^{(j)}\right]=\varepsilon_{\boldsymbol{k} 0}^{(i)}+\sum_{\boldsymbol{k}^{\prime} \sigma^{\prime} j} f^{i j}\left(\boldsymbol{k}, \boldsymbol{k}^{\prime}\right)\left[\mathcal{N}_{\boldsymbol{k}^{\prime}}^{(j)}-\theta_{\boldsymbol{k}^{\prime}}^{(j)}\right] .
$$

For nonsuperfluid matter in thermodynamic equilibrium the distribution function $\mathcal{N}_{\boldsymbol{k}}^{(i)}$ is denoted $n_{\boldsymbol{k} 0}^{(i)}$ and is given by

$$
n_{\boldsymbol{k} 0}^{(i)}=\left\langle a_{\boldsymbol{k} \sigma}^{(i) \dagger} a_{\boldsymbol{k} \sigma}^{(i)}\right\rangle=\frac{1}{1+\mathrm{e}^{\left(\varepsilon_{\boldsymbol{k}}^{(i)}\left[n_{\boldsymbol{k} 0}^{(j)}\right]-\mu_{i}\right) / T}}
$$

In Eqs. (1) and (2) $\varepsilon_{\boldsymbol{k} 0}^{(i)}$ is the quasiparticle energy at $T=0 ; \sigma$ and $\sigma^{\prime}= \pm 1$ are spin indices; $\theta_{\boldsymbol{k}}^{(i)}=\theta\left(k_{\mathrm{F} i}-k\right)$, where $\theta(x)$ is the step function, and $k_{\mathrm{F} i}$ is the Fermi momentum. Furthermore, $a_{\boldsymbol{k} \sigma}^{(i)}=a_{\boldsymbol{k} \uparrow}^{(i)}$ or $a_{\boldsymbol{k} \downarrow}^{(i)}$ is the annihilation operator of a quasiparticle in a state $(\boldsymbol{k} \sigma)$ and $\mu_{i}$ is the chemical potential. Finally, $f^{i j}\left(\boldsymbol{k}, \boldsymbol{k}^{\prime}\right)$ in Eq. (1) is the Landau quasiparticle interaction. In the present paper we only deal with the spin-unpolarized matter and do not consider forces that rotate spin. This allows us to disregard the spin dependence of the interaction function, assuming that $f^{i j}\left(\boldsymbol{k}, \boldsymbol{k}^{\prime}\right)$ is already averaged over spin variables. We also suppress spin indices in formulas, whenever possible. Notice, however, that the kinetic equation (19), obtained below, can be used, with minor modifications, to study transport properties of spin-polarized matter [then, of course, one should take into account the spin-dependence of $f^{i j}\left(\boldsymbol{k}, \boldsymbol{k}^{\prime}\right)$ ].

In the vicinity of the Fermi surface the lengths of the vectors $\boldsymbol{k}$ and $\boldsymbol{k}^{\prime}$ in the arguments of the function $f^{i j}\left(\boldsymbol{k}, \boldsymbol{k}^{\prime}\right)$ can be approximately set equal to $k \approx k_{\mathrm{F} i}$ and $k^{\prime} \approx k_{\mathrm{F} j}$, while the function itself can be expanded into Legendre polynomials $P_{l}(\cos \theta)$,

$$
f^{i j}\left(\boldsymbol{k}, \boldsymbol{k}^{\prime}\right)=\sum_{l} f_{l}^{i j} P_{l}(\cos \theta)
$$


where $\theta$ is the angle between $\boldsymbol{k}$ and $\boldsymbol{k}^{\prime}$, and $f_{l}^{i j}$ are the symmetric Landau parameters, $f_{l}^{i j}=f_{l}^{j i}$.

Assume now that both particles, $i=1$ and $i=2$, are superfluid (and we are still in thermodynamic equilibrium at a temperature $T$ ). The spin $1 / 2$ elementary excitations in superfluid Fermi-liquid are the Bogoliubov excitations. In the absence of superfluid currents in the system, their energy $E_{\boldsymbol{k}}^{(i)}$ and the distribution function $\mathcal{F}_{\boldsymbol{k} 0}^{(i)}$ are (see, e.g., Ref. [47])

$$
\begin{aligned}
E_{\boldsymbol{k}}^{(i)} & =\sqrt{\xi_{\boldsymbol{k}}^{(i) 2}+\Delta_{i}^{2}}, \\
\mathcal{F}_{\boldsymbol{k} 0}^{(i)} & =\frac{1}{1+\mathrm{e}^{E_{\boldsymbol{k}}^{(i)} / T}},
\end{aligned}
$$

where $\xi_{\boldsymbol{k}}^{(i)}$ equals (see, e.g., Ref. [38])

$$
\xi_{\boldsymbol{k}}^{(i)}=\varepsilon_{\boldsymbol{k}}^{(i)}\left[\mathcal{N}_{\boldsymbol{k} 0}^{(j)}\right]-\mu_{i}=v_{\mathrm{F} i}\left(k-k_{\mathrm{F} i}\right)+O\left[\left(\frac{T}{\mu_{j}}\right)^{2}+\left(\frac{\Delta_{i}}{\mu_{j}}\right)^{2}\right] \approx v_{\mathrm{F} i}\left(k-k_{\mathrm{F} i}\right) .
$$

The function $\mathcal{N}_{k 0}^{(i)}$ will be defined in Eq. (16); $v_{\mathrm{F} i}=k_{\mathrm{F} i} / m_{i}^{*}$ is the Fermi velocity and $m_{i}^{*}$ is the effective mass. In the nonrelativistic limit $m_{i}^{*}$ can be found from the equation [37, 38, 48]

$$
\frac{m_{i}}{m_{i}^{*}}=1-\sum_{j} \frac{m_{j} G_{i j}}{n_{i}}
$$

where

$$
n_{i}=\frac{p_{\mathrm{F} i}^{3}}{3 \pi^{2}}
$$

is the number density; $m_{i}$ is the bare mass; and the symmetric matrix $G_{i j}$ is defined as

$$
G_{i j} \equiv \frac{1}{9 \pi^{4}} p_{\mathrm{F} i}^{2} p_{\mathrm{F} j}^{2} f_{1}^{i j}
$$

Furthermore, $\Delta_{i}$ in Eqs. (44)-(6) is the energy gap in thermodynamic equilibrium. Because we assume the singletstate ${ }^{1} \mathrm{~S}_{0}$ pairing of quasiparticles, the gap $\Delta_{i}$ depends only on $k=|\boldsymbol{k}|$. It can be found from the standard equation

$$
\Delta_{i}(k)=-\sum_{\boldsymbol{k}^{\prime}} V^{(i)}\left(\boldsymbol{k}, \boldsymbol{k}^{\prime}\right) \Delta_{i}\left(k^{\prime}\right) F_{\boldsymbol{k}^{\prime}}^{(i)} .
$$

Here we define

$$
F_{\boldsymbol{k}}^{(i)} \equiv \frac{1}{2 E_{\boldsymbol{k}}^{(i)}} \tanh \left(\frac{E_{\boldsymbol{k}}^{(i)}}{2 T}\right) .
$$

In Eq. (10) $V^{(i)}\left(\boldsymbol{k}, \boldsymbol{k}^{\prime}\right)$ is the pairing potential for particles $i$. In analogy with the function $f^{i j}\left(\boldsymbol{k}, \boldsymbol{k}^{\prime}\right)$ [see Eq. (3)], we expand it into Legendre polynomials,

$$
V^{(i)}\left(\boldsymbol{k}, \boldsymbol{k}^{\prime}\right)=\sum_{l} V_{l}^{(i)} P_{l}(\cos \theta)
$$

Near the Fermi surface a smoothly varying function $\Delta_{i}(k)$ can be approximated as $\Delta_{i}(k) \approx \Delta_{i}\left(k_{\mathrm{F} i}\right)$ (see, e.g., Ref. [24]). Then, combining Eqs. (10) and (12), one obtains the following equation for $\Delta_{i}$,

$$
1=-V_{0}^{(i)} \sum_{\boldsymbol{k}^{\prime}} F_{\boldsymbol{k}^{\prime}}^{(i)}
$$

As follows from Eq. (5), the distribution function $\mathcal{F}_{\boldsymbol{k} 0}^{(i)}$ for Bogoliubov excitations is a scalar quantity. In contrast, the distribution function $\mathbf{n}_{\boldsymbol{k} \sigma 0}^{(i)}$ for Landau quasiparticles in superfluid matter is a matrix rather than a scalar. In thermodynamic equilibrium it can be written as (see, e.g., Ref. [24])

$$
\mathbf{n}_{\boldsymbol{k} \sigma 0}^{(i)}=\left(\begin{array}{cc}
\left\langle a_{\boldsymbol{k} \sigma}^{(i) \dagger} a_{\boldsymbol{k} \sigma}^{(i)}\right\rangle & \left\langle a_{\boldsymbol{k} \sigma}^{(i) \dagger} a_{-\boldsymbol{k}-\sigma}^{(i) \dagger}\right\rangle \\
\left\langle a_{-\boldsymbol{k}-\sigma}^{(i)} a_{\boldsymbol{k} \sigma}^{(i)}\right\rangle & \left\langle a_{-\boldsymbol{k}-\sigma}^{(i)} a_{-\boldsymbol{k}-\sigma}^{(i) \dagger}\right\rangle
\end{array}\right)=\frac{1}{2}\left(\hat{\mathbf{1}}-2 \boldsymbol{\epsilon}_{\boldsymbol{k} \sigma 0}^{(i)} F_{\boldsymbol{k}}^{(i)}\right),
$$


where $\hat{\mathbf{1}}$ is the unit matrix. The 'energy matrix' $\boldsymbol{\epsilon}_{\boldsymbol{k} \sigma 0}^{(i)}$ in Eq. (14) equals

$$
\boldsymbol{\epsilon}_{\boldsymbol{k} \sigma 0}^{(i)}=\left(\begin{array}{cc}
\xi_{\boldsymbol{k}}^{(i)} & \sigma \Delta_{i} \\
\sigma \Delta_{i} & -\xi_{\boldsymbol{k}}^{(i)}
\end{array}\right)
$$

For superfluid matter in the absence of superfluid currents the average equilibrium number $\mathcal{N}_{\boldsymbol{k} 0}^{(i)}$ of Landau quasiparticles in a state $(\boldsymbol{k} \sigma)$ is given by the element $\left\langle a_{\boldsymbol{k} \sigma}^{(i) \dagger} a_{\boldsymbol{k} \sigma}^{(i)}\right\rangle$ of the matrix $\mathbf{n}_{\boldsymbol{k} \sigma 0}^{(i)}$,

$$
\mathcal{N}_{\boldsymbol{k} 0}^{(i)} \equiv\left\langle a_{\boldsymbol{k} \sigma}^{(i) \dagger} a_{\boldsymbol{k} \sigma}^{(i)}\right\rangle=\frac{1}{2}\left(1-2 \xi_{\boldsymbol{k}}^{(i)} F_{\boldsymbol{k}}^{(i)}\right)
$$

[compare this expression with the corresponding Eq. (2) for normal matter].

\section{B. The system of kinetic equations}

To obtain the kinetic equation let us slightly perturb the system. Since our aim in this section is to determine the kinetic equation in the linear approximation, we may assume, without any loss of generality, that the perturbation varies with coordinate $\boldsymbol{r}$ and time $t$ as $\mathrm{e}^{i(\boldsymbol{q} \boldsymbol{r}-\omega t)}$, where $\boldsymbol{q}$ and $\omega$ are the perturbation wave vector and frequency, respectively. To use the Landau theory of Fermi-liquids we have to assume, in addition, that $q \ll k_{\mathrm{F} i}$ and $\omega \ll \mu_{i}$. [24])

The only non-zero matrix elements, induced by the perturbation, can be written in a compact form as (see, e.g.,

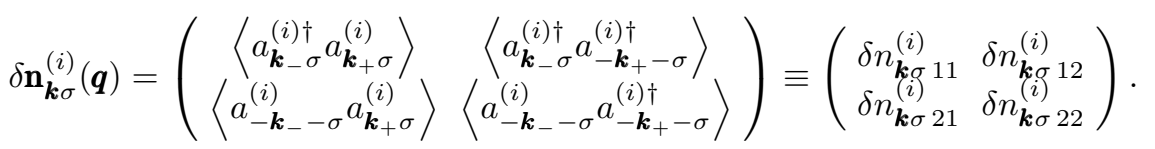

Here and below we use the notation

$$
k_{-}=k-\frac{q}{2}, \quad k_{+}=k+\frac{q}{2}
$$

The matrix $\delta \mathbf{n}_{\boldsymbol{k} \sigma}^{(i)}(\boldsymbol{q})$ with the elements $\delta n_{\boldsymbol{k} \sigma 11}^{(i)}, \ldots, \delta n_{\boldsymbol{k} \sigma 22}^{(i)}$, defined in Eq. (17), can be interpreted as a small deviation from the equilibrium distribution function $\mathbf{n}_{\boldsymbol{k} \sigma 0}^{(i)}$, caused by the perturbation. The collisionless kinetic equation for $\delta \mathbf{n}_{\boldsymbol{k} \sigma}^{(i)}(\boldsymbol{q})$ can be found following the derivation of Ref. [24]. The result is:

$$
\omega \delta \mathbf{n}_{\boldsymbol{k} \sigma}^{(i)}=\delta \mathbf{n}_{\boldsymbol{k} \sigma}^{(i)} \boldsymbol{\epsilon}_{\boldsymbol{k}_{+} \sigma 0}^{(i)}-\boldsymbol{\epsilon}_{\boldsymbol{k}_{-\sigma 0}}^{(i)} \delta \mathbf{n}_{\boldsymbol{k} \sigma}^{(i)}+\mathbf{n}_{\boldsymbol{k}_{-\sigma 0}}^{(i)} \delta \boldsymbol{\epsilon}_{\boldsymbol{k} \sigma}^{(i)}-\delta \boldsymbol{\epsilon}_{\boldsymbol{k} \sigma}^{(i)} \mathbf{n}_{\boldsymbol{k}_{+} \sigma 0}^{(i)} .
$$

In this equation $\delta \boldsymbol{\epsilon}_{\boldsymbol{k} \sigma}^{(i)}$ is a matrix, describing local deviation of the quasiparticle energy from its equilibrium value $\boldsymbol{\epsilon}_{\boldsymbol{k} \sigma 0}^{(i)}$. It is the sum of two terms,

$$
\delta \boldsymbol{\epsilon}_{\boldsymbol{k} \sigma}^{(i)}=\lambda_{\boldsymbol{k} \sigma}^{(i)}+\Lambda_{\boldsymbol{k} \sigma}^{(i)}
$$

where the term $\lambda_{\boldsymbol{k} \sigma}^{(i)}$ describes the change of the quasiparticle energy with the distribution function,

$$
\lambda_{\boldsymbol{k} \sigma}^{(i)}=\left(\begin{array}{ccc}
\sum_{\boldsymbol{k}^{\prime} \sigma^{\prime} j} f^{i j}\left(\boldsymbol{k}, \boldsymbol{k}^{\prime}\right) \delta n_{\boldsymbol{k}^{\prime} \sigma^{\prime} 11}^{(j)} & \sum_{\boldsymbol{k}^{\prime}} V^{(i)}\left(\boldsymbol{k}, \boldsymbol{k}^{\prime}\right) \delta n_{\boldsymbol{k}^{\prime} \sigma 12}^{(i)} \\
\sum_{\boldsymbol{k}^{\prime}} V^{(i)}\left(\boldsymbol{k}, \boldsymbol{k}^{\prime}\right) \delta n_{\boldsymbol{k}^{\prime} \sigma 21}^{(i)} & \sum_{\boldsymbol{k}^{\prime} \sigma^{\prime} j} f^{i j}\left(\boldsymbol{k}, \boldsymbol{k}^{\prime}\right) \delta n_{\boldsymbol{k}^{\prime} \sigma^{\prime} 22}^{(j)}
\end{array}\right),
$$

and the term $\Lambda_{\boldsymbol{k} \sigma}^{(i)}$ is responsible for the interaction of quasiparticles with the self-consistent electromagnetic field,

$$
\Lambda_{\boldsymbol{k} \sigma}^{(i)}=\left(\begin{array}{cc}
e_{i} V-\alpha_{i} \frac{\boldsymbol{k A}}{m_{i}} & 0 \\
0 & -e_{i} V-\alpha_{i} \frac{\boldsymbol{k A}}{m_{i}}
\end{array}\right)
$$

Here $V$ and $\boldsymbol{A}$ are the scalar and vector electromagnetic potentials, respectively. It is assumed that in the unperturbed system $V=0$ and $\boldsymbol{A}=0$. 
Equations (19)-(22) are trivial generalizations, to the case of superfluid mixtures, of the corresponding equations presented in Ref. [24]. The only non-trivial point is the expression for the coefficient $\alpha_{i}$. In Ref. [24] $\alpha_{i}$ is equal to the quasiparticle electric charge $e_{i}$. This result is valid only for a one-component Fermi-liquid. As is demonstrated in the Appendix A, for a mixture of Fermi-liquids one should instead write

$$
\alpha_{i}=\frac{m_{i}}{n_{i}} \sum_{j} e_{j} Y_{i j}
$$

where $Y_{i j}$ is the relativistic entrainment matrix at zero temperature, given by [48, 49]

$$
Y_{i j}=\frac{n_{i}}{m_{i}^{*}} \delta_{i j}+G_{i j}
$$

In this equation $\delta_{i j}$ is the Kronecker symbol and the matrix $G_{i j}$ is defined in Eq. (99).

The kinetic equation (19) consists of four coupled integral equations. Their solution determines the matrix $\delta \mathbf{n}_{\boldsymbol{k} \sigma}^{(i)}(\boldsymbol{q})$ [i.e., the four functions $\delta n_{\boldsymbol{k} \sigma 11}^{(i)}, \ldots, \delta n_{\boldsymbol{k} \sigma 22}^{(i)}$ ].

The system (19) can be substantially simplified by introducing a new set of variables

$$
\begin{aligned}
\delta n_{\boldsymbol{k} \sigma+}^{(i)} & =\delta n_{\boldsymbol{k} \sigma 11}^{(i)}+\delta n_{\boldsymbol{k} \sigma 22}^{(i)}, \\
\delta n_{\boldsymbol{k} \sigma-}^{(i)} & =\delta n_{\boldsymbol{k} \sigma 11}^{(i)}-\delta n_{\boldsymbol{k} \sigma 22}^{(i)}, \\
\delta s_{\boldsymbol{k} \sigma+}^{(i)} & =\sigma\left[\delta n_{\boldsymbol{k} \sigma 12}^{(i)}+\delta n_{\boldsymbol{k} \sigma 21}^{(i)}\right], \\
\delta s_{\boldsymbol{k} \sigma-}^{(i)} & =\sigma\left[\delta n_{\boldsymbol{k} \sigma 12}^{(i)}-\delta n_{\boldsymbol{k} \sigma 21}^{(i)}\right]
\end{aligned}
$$

with the obvious symmetry properties [see Eq. (17)],

$$
\begin{aligned}
\delta n_{\boldsymbol{k} \sigma+}^{(i)} & =-\delta n_{-\boldsymbol{k}-\sigma+}^{(i)}, \\
\delta n_{\boldsymbol{k} \sigma-}^{(i)} & =\delta n_{-\boldsymbol{k}-\sigma-}^{(i)}, \\
\delta s_{\boldsymbol{k} \sigma+}^{(i)} & =\delta s_{-\boldsymbol{k}-\sigma+}^{(i)}, \\
\delta s_{\boldsymbol{k} \sigma-}^{(i)} & =\delta s_{-\boldsymbol{k}-\sigma-}^{(i)}
\end{aligned}
$$

Using these variables, the system of kinetic equations (19) can be rewritten in the form

$$
\begin{aligned}
& \omega \delta n_{\boldsymbol{k}_{\sigma+}}^{(i)}=\left[F_{\boldsymbol{k}_{+}}^{(i)} \xi_{\boldsymbol{k}_{+}}^{(i)}-F_{\boldsymbol{k}_{-}}^{(i)} \xi_{\boldsymbol{k}_{-}}^{(i)}\right] V_{\boldsymbol{k}}^{(i)}+\left[\xi_{\boldsymbol{k}_{+}}^{(i)}-\xi_{\boldsymbol{k}_{-}}^{(i)}\right] \delta n_{\boldsymbol{k} \sigma-}^{(i)} \\
& +\Delta_{i}\left[F_{\boldsymbol{k}_{+}}^{(i)}-F_{\boldsymbol{k}_{-}}^{(i)}\right] O_{\boldsymbol{k} \sigma+}^{(i)}, \\
& \omega \delta n_{\boldsymbol{k}_{\sigma-}}^{(i)}=\left[F_{\boldsymbol{k}_{-}}^{(i)} \xi_{\boldsymbol{k}_{-}}^{(i)}-F_{\boldsymbol{k}_{+}}^{(i)} \xi_{\boldsymbol{k}_{+}}^{(i)}\right] A_{\boldsymbol{k}}^{(i)}+\left[\xi_{\boldsymbol{k}_{+}}^{(i)}-\xi_{\boldsymbol{k}_{-}}^{(i)}\right] \delta n_{\boldsymbol{k} \sigma+}^{(i)} \\
& +2 \Delta_{i} \delta s_{\boldsymbol{k} \sigma-}^{(i)}+\Delta_{i}\left[F_{\boldsymbol{k}_{-}}^{(i)}+F_{\boldsymbol{k}_{+}}^{(i)}\right] O_{\boldsymbol{k} \sigma-}^{(i)}, \\
& \omega \delta s_{\boldsymbol{k} \sigma-}^{(i)}=\Delta_{i}\left[F_{\boldsymbol{k}_{-}}^{(i)}+F_{\boldsymbol{k}_{+}}^{(i)}\right] V_{\boldsymbol{k} \sigma}^{(i)}+2 \Delta_{i} \delta n_{\boldsymbol{k} \sigma-}^{(i)} \\
& -\left[\xi_{\boldsymbol{k}_{-}}^{(i)}+\xi_{\boldsymbol{k}_{+}}^{(i)}\right] \delta s_{\boldsymbol{k} \sigma+}^{(i)}-\left[F_{\boldsymbol{k}_{-}}^{(i)} \xi_{\boldsymbol{k}_{-}}^{(i)}+F_{\boldsymbol{k}_{+}}^{(i)} \xi_{\boldsymbol{k}_{+}}^{(i)}\right] O_{\boldsymbol{k} \sigma+}^{(i)}, \\
& \omega \delta s_{\boldsymbol{k} \sigma+}^{(i)}=\Delta_{i}\left[F_{\boldsymbol{k}_{-}}^{(i)}-F_{\boldsymbol{k}_{+}}^{(i)}\right] A_{\boldsymbol{k} \sigma}^{(i)} \\
& -\left[\xi_{\boldsymbol{k}_{-}}^{(i)}+\xi_{\boldsymbol{k}_{+}}^{(i)}\right] \delta s_{\boldsymbol{k} \sigma-_{-}}^{(i)}-\left[F_{\boldsymbol{k}_{-}}^{(i)} \xi_{\boldsymbol{k}_{-}}^{(i)}+F_{\boldsymbol{k}_{+}}^{(i)} \xi_{\boldsymbol{k}_{+}}^{(i)}\right] O_{\boldsymbol{k} \sigma-}^{(i)},
\end{aligned}
$$

where $O_{\boldsymbol{k} \sigma+}^{(i)}$ and $O_{\boldsymbol{k} \sigma-}^{(i)}$ equal

$$
\begin{aligned}
& O_{\boldsymbol{k} \sigma+}^{(i)}=\sum_{\boldsymbol{k}^{\prime}} V^{(i)}\left(\boldsymbol{k}, \boldsymbol{k}^{\prime}\right) \delta s_{\boldsymbol{k}^{\prime} \sigma+}^{(i)}, \\
& O_{\boldsymbol{k} \sigma-}^{(i)}=\sum_{\boldsymbol{k}^{\prime}} V^{(i)}\left(\boldsymbol{k}, \boldsymbol{k}^{\prime}\right) \delta s_{\boldsymbol{k}^{\prime} \sigma-}^{(i)},
\end{aligned}
$$


and the functions $V_{\boldsymbol{k} \sigma}^{(i)}$ and $A_{\boldsymbol{k} \sigma}^{(i)}$ are defined as

$$
\begin{aligned}
V_{\boldsymbol{k} \sigma}^{(i)} & =2 e_{i} V+\sum_{\boldsymbol{k}^{\prime} \sigma^{\prime} j} f^{i j}\left(\boldsymbol{k}, \boldsymbol{k}^{\prime}\right) \delta n_{\boldsymbol{k}^{\prime} \sigma^{\prime}-}^{(j)} \\
A_{\boldsymbol{k} \sigma}^{(i)} & =2 \alpha_{i} \frac{\boldsymbol{k} \boldsymbol{A}}{m_{i}}-\sum_{\boldsymbol{k}^{\prime} \sigma^{\prime} j} f^{i j}\left(\boldsymbol{k}, \boldsymbol{k}^{\prime}\right) \delta n_{\boldsymbol{k}^{\prime} \sigma^{\prime}+}^{(j)} .
\end{aligned}
$$

The system (33)-(36) contains all information to calculate linear gauge-invariant response of the two-component superfluid Fermi-liquid to a vector (e.g., electromagnetic) perturbation.

\section{The particle current density}

Let us sum Eq. (34) over $\boldsymbol{k}$ and $\sigma$. Then, using Eq. (13) one obtains that, with the accuracy up to quadratic terms in $q / k_{\mathrm{F} i} \ll 1$, the last two terms in the right-hand side of Eq. (34) cancel out and we are left with

$$
\omega \sum_{\boldsymbol{k} \sigma} \delta n_{\boldsymbol{k} \sigma-}^{(i)}=\sum_{\boldsymbol{k} \sigma}\left[\mathcal{N}_{\boldsymbol{k}_{+} 0}^{(i)}-\mathcal{N}_{\boldsymbol{k}_{-} 0}^{(i)}\right] A_{\boldsymbol{k} \sigma}^{(i)}+\sum_{\boldsymbol{k} \sigma}\left[\xi_{\boldsymbol{k}_{+}}^{(i)}-\xi_{\boldsymbol{k}_{-}}^{(i)}\right] \delta n_{\boldsymbol{k} \sigma+}^{(i)} \cdot
$$

Here we also made use of Eq. (16). To proceed further, we expand $\xi_{\boldsymbol{k}_{ \pm}}^{(i)}$ in Taylor series,

$$
\xi_{\boldsymbol{k}_{ \pm}}^{(i)}=\xi_{\boldsymbol{k}}^{(i)} \pm \frac{\boldsymbol{q} \boldsymbol{v}_{i}}{2}+O\left[\left(\frac{q}{k_{\mathrm{F} i}}\right)^{2}\right]
$$

where $\boldsymbol{v}_{i}$ is the velocity of Landau quasiparticles on the Fermi surface, $\boldsymbol{v}_{i} \equiv v_{\mathrm{F} i}(\boldsymbol{k} / k)$.

Eq. (41) can be additionally simplified with the help of Eq. (40) and the expansion (42). Neglecting all terms of the second and higher orders in $q / k_{\mathrm{F} i}$, one obtains particle conservation law

$$
\omega \delta n_{i}=\boldsymbol{q} \boldsymbol{j}_{i}
$$

with

$$
\begin{aligned}
\delta n_{i} & =\frac{1}{2} \sum_{\boldsymbol{k} \sigma} \delta n_{\boldsymbol{k} \sigma-}^{(i)}, \\
\boldsymbol{j}_{i} & =\frac{1}{2} \sum_{\boldsymbol{k} \sigma}\left[\boldsymbol{v}_{i} \delta n_{\boldsymbol{k} \sigma+}^{(i)}+\mathcal{N}_{\boldsymbol{k} 0}^{(i)} \frac{\partial}{\partial \boldsymbol{k}} \sum_{\boldsymbol{k}^{\prime} \sigma^{\prime} j} f^{i j}\left(\boldsymbol{k}, \boldsymbol{k}^{\prime}\right) \delta n_{\boldsymbol{k}^{\prime} \sigma^{\prime}+}^{(j)}\right]-\alpha_{i} \frac{n_{i}}{m_{i}} \boldsymbol{A} .
\end{aligned}
$$

Because $\mathcal{N}_{\boldsymbol{k} 0}^{(i)}$ is the isotropic equilibrium distribution function, one has $\partial \mathcal{N}_{\boldsymbol{k} 0}^{(i)} / \partial \boldsymbol{k}=\left[\partial \mathcal{N}_{\boldsymbol{k} 0}^{(i)} / \partial k\right](\boldsymbol{k} / k)$, so that Eq. (45) can be rewritten as

$$
\boldsymbol{j}_{i}=\frac{1}{2} \sum_{\boldsymbol{k} \sigma}\left[\boldsymbol{v}_{i} \delta n_{\boldsymbol{k} \sigma+}^{(i)}-\sum_{\boldsymbol{k}^{\prime} \sigma^{\prime} j} \frac{\partial \mathcal{N}_{\boldsymbol{k} 0}^{(i)}}{\partial k} f_{1}^{i j} \frac{\boldsymbol{k}\left(\boldsymbol{k} \boldsymbol{k}^{\prime}\right)}{k^{2} k^{\prime}} \delta n_{\boldsymbol{k}^{\prime} \sigma^{\prime}+}^{(j)}\right]-\alpha_{i} \frac{n_{i}}{m_{i}} \boldsymbol{A} .
$$

where the second term in square brackets was integrated by parts and we made use of the expansion (3).

The quantities $\delta n_{i}$ and $\boldsymbol{j}_{i}$ in Eqs. (43)-(46) can be interpreted as the number density perturbation and particle current density, respectively. To prove that this is really so, we employ Eqs. (25) and (26) and the symmetry relations (29) and (30), and rewrite Eqs. (44) and (45) in the familiar form,

$$
\begin{aligned}
\delta n_{i} & =\sum_{\boldsymbol{k} \sigma} \delta n_{\boldsymbol{k} \sigma 11}^{(i)}, \\
\boldsymbol{j}_{i} & =\sum_{\boldsymbol{k} \sigma}\left[\boldsymbol{v}_{i} \delta n_{\boldsymbol{k} \sigma 11}^{(i)}+\mathcal{N}_{\boldsymbol{k} 0}^{(i)} \frac{\partial}{\partial \boldsymbol{k}} \sum_{\boldsymbol{k}^{\prime} \sigma^{\prime} j} f^{i j}\left(\boldsymbol{k}, \boldsymbol{k}^{\prime}\right) \delta n_{\boldsymbol{k}^{\prime} \sigma^{\prime} 11}^{(j)}\right]-\alpha_{i} \frac{n_{i}}{m_{i}} \boldsymbol{A} .
\end{aligned}
$$


One sees that $\delta n_{i}$ is indeed the number density perturbation. Thus, as follows from the continuity equation (43), $\boldsymbol{j}_{i}$ is the particle current density. Notice that, $\boldsymbol{j}_{i}$ is formally given by the same expression as for normal (nonsuperfluid) Fermi-liquid (see, e.g., Refs. [45, 46]). For a one-component Fermi-liquid this was first demonstrated by Leggett [44].

Since the quantities $\delta n_{i}$ and $\boldsymbol{j}_{i}$ are observables, they must be invariant under gauge transformations. This property of $\delta n_{i}$ and $\boldsymbol{j}_{i}$ is very important for the consideration below. For a one-component Fermi-liquid the gauge invariance of $\delta n_{i}$ and $\boldsymbol{j}_{i}$, defined in Eqs. (44)-(48), was explicitly demonstrated by Betbeder-Matibet and Nozieres [24] [see their Eq. (28)].

\section{LANDAU FERMI-LIQUID EFFECTS AND GAUGE INVARIANCE}

In this section we study the response of the system to an applied harmonic electromagnetic field. Our aim will be to express the exact polarization tensor for a strongly interacting two-component Fermi-liquid through that of noninteracting liquid [for which the Landau quasiparticle interaction $f^{i j}\left(\boldsymbol{k}, \boldsymbol{k}^{\prime}\right)=0$ ].

\section{A. Gauge-invariant expressions for $\delta n_{i}$ and $\boldsymbol{j}_{i}$}

Assume that $V$ and $\boldsymbol{A}$ are some self-consistent (not external) vector and scalar electromagnetic potentials in an arbitrary gauge. The vector potential $\boldsymbol{A}$ can be presented in the form

$$
A=A_{\mathrm{l}}+A_{\text {tr }},
$$

where $\boldsymbol{A}_{1}=\boldsymbol{q}(\boldsymbol{q} \boldsymbol{A}) / q^{2}$ is the longitudinal component of $\boldsymbol{A}$ directed along $\boldsymbol{q}$ and $\boldsymbol{A}_{\mathrm{tr}}=\boldsymbol{A}-\boldsymbol{A}_{\mathrm{l}}$ is the transverse component.

Performing a gauge transformation

$$
\begin{aligned}
& \widetilde{V}=V+\frac{\partial \phi}{\partial t}=V-i \omega \phi, \\
& \widetilde{\boldsymbol{A}}=\boldsymbol{A}-\boldsymbol{\nabla} \phi=\boldsymbol{A}-i \boldsymbol{q} \phi,
\end{aligned}
$$

one can choose the new potentials $\widetilde{V}$ and $\widetilde{\boldsymbol{A}}$ in such a way that $\widetilde{\boldsymbol{A}}_{1}=0$ (i.e. $\phi=-i A_{1} / q$ ). In this new gauge the relation between $\delta n_{i}, \boldsymbol{j}_{i}$ and $\widetilde{V}, \widetilde{A}$ can generally be written as

$$
\begin{aligned}
\delta n_{i} & =P_{00}^{(i)} \widetilde{V} \\
\boldsymbol{j}_{i} & =P_{\mathrm{tr}}^{(i)} \widetilde{\boldsymbol{A}}_{\mathrm{tr}}+\frac{\boldsymbol{q}}{\omega} P_{1}^{(i)} \widetilde{V},
\end{aligned}
$$

where $P_{1}^{(i)}$ and $P_{\mathrm{tr}}^{(i)}$ are, respectively, the exact longitudinal and transverse polarization functions for a strongly interacting Fermi-mixture. The function $P_{00}^{(i)}$ is related to $P_{1}^{(i)}$ by the continuity equation (43),

$$
P_{1}^{(i)}=\frac{\omega^{2}}{q^{2}} P_{00}^{(i)}
$$

A direct (but not easy) way to obtain the quantities $P_{00}^{(i)}, P_{1}^{(i)}$, and $P_{\mathrm{tr}}^{(i)}$ is to solve the system of integral kinetic equations (33)-(36) for $\delta n_{\boldsymbol{k} \sigma-}^{(i)}$ and $\delta n_{\boldsymbol{k} \sigma+}^{(i)}$, and then to make use of Eqs. (44) and (45).

Employing Eqs. (50) and (51), one can rewrite Eqs. (52) and (53) in the original gauge,

$$
\begin{aligned}
\delta n_{i} & =P_{00}^{(i)}\left(V-\frac{\omega}{q} A_{1}\right), \\
\boldsymbol{j}_{i} & =P_{\mathrm{tr}}^{(i)} A_{\mathrm{tr}}+\frac{\boldsymbol{q}}{\omega} P_{1}^{(i)}\left(V-\frac{\omega}{q} A_{1}\right) .
\end{aligned}
$$

Clearly, these expressions are gauge-invariant. The knowledge of $P_{00}^{(i)}, P_{1}^{(i)}$, and $P_{\mathrm{tr}}^{(i)}$ allows one to determine the longitudinal $\varepsilon_{1}$ and transverse $\varepsilon_{\text {tr }}$ dielectric functions of the liquid,

$$
\begin{aligned}
\varepsilon_{1} & =1-\frac{4 \pi}{\omega^{2}} \sum_{i} e_{i} P_{1}^{(i)}=1-\frac{4 \pi}{q^{2}} \sum_{i} e_{i} P_{00}^{(i)}, \\
\varepsilon_{\mathrm{tr}} & =1-\frac{4 \pi}{\omega^{2}} \sum_{i} e_{i} P_{\mathrm{tr}}^{(i)} .
\end{aligned}
$$


For noninteracting Fermi-liquid, for which $f^{i j}\left(\boldsymbol{k}, \boldsymbol{k}^{\prime}\right)=0$, Eqs. (55) and (56) take the form

$$
\begin{aligned}
\delta n_{i} & =\Pi_{00}^{(i)}\left(V-\frac{\omega}{q} A_{\mathrm{l}}\right), \\
\boldsymbol{j}_{i} & =\Pi_{\mathrm{tr}}^{(i)} A_{\mathrm{tr}}+\frac{\boldsymbol{q}}{\omega} \Pi_{\mathrm{l}}^{(i)}\left(V-\frac{\omega}{q} A_{\mathrm{l}}\right),
\end{aligned}
$$

where the exact polarization functions of noninteracting matter are denoted as $\Pi_{00}^{(i)}, \Pi_{1}^{(i)}$, and $\Pi_{\text {tr }}^{(i)}$. These quantities were carefully analyzed in the literature (see, e.g., Refs. [9, 50 52]); they depend on a number of parameters, in particular, on the particle Fermi-momentum $p_{\mathrm{F} i}$ and on the mass $m_{i}$.

\section{B. Landau quasiparticle interaction and the polarization functions}

Let us calculate the exact polarization functions $P_{00}^{(i)}, P_{1}^{(i)}$, and $P_{\mathrm{tr}}^{(i)}$ under the simplified assumption that all the Landau parameters except for $f_{0}^{i j}$ and $f_{1}^{i j}$ vanish, $f_{l}^{i j}=0$ for $l \geq 2$. Eqs. (39) and (40) can then be rewritten as

$$
\begin{aligned}
V_{\boldsymbol{k} \sigma}^{(i)} & =2 e_{i} V+2 \sum_{j} f_{0}^{i j} \delta n_{j} \equiv 2 e_{i} V_{\mathrm{eff}}^{(i)}, \\
A_{\boldsymbol{k} \sigma}^{(i)} & =2 \alpha_{i} \frac{\boldsymbol{k} \boldsymbol{A}}{m_{i}}-\sum_{j} \frac{\boldsymbol{k}}{k_{\mathrm{F} i} k_{\mathrm{F} j}} f_{1}^{i j} \sum_{\boldsymbol{k}^{\prime} \sigma^{\prime}} \boldsymbol{k}^{\prime} \delta n_{\boldsymbol{k}^{\prime} \sigma^{\prime}+}^{(j)} \equiv 2 e_{i} \frac{\boldsymbol{k} \boldsymbol{A}_{\mathrm{eff}}^{(i)}}{m_{i}^{*}},
\end{aligned}
$$

where we employed Eqs. (29), (30), (44), and introduced the effective scalar $V_{\text {eff }}^{(i)}$ and vector $A_{\text {eff }}^{(i)}$ electromagnetic potentials. One can express them in terms of real electromagnetic potentials $V$ and $\boldsymbol{A}$ and gauge-invariant observables $\delta n_{i}$ and $\boldsymbol{j}_{i}$ by making use of Eqs. (44) and (45), respectively. The result is:

$$
\begin{aligned}
& V_{\mathrm{eff}}^{(i)}=V+\frac{1}{e_{i}} \sum_{j} f_{0}^{i j} \delta n_{j}, \\
& \boldsymbol{A}_{\mathrm{eff}}^{(i)}=\boldsymbol{A}+\frac{1}{e_{i}} \sum_{j} \gamma_{i j} j_{j} .
\end{aligned}
$$

To obtain Eq. (64) we use Eq. (7) for the effective mass $m_{i}^{*}$ and the expression (23) for $\alpha_{i}$. The detailed derivation of Eq. (64) for a one-component Fermi-liquid is given in Appendix B. The matrix $\gamma_{i j}$ in Eq. (64) depends on the Landau parameters $f_{1}^{i j}$ and equals

$$
\begin{aligned}
\gamma_{i i} & =\frac{m_{i}^{2}}{S_{i}}\left(G_{i i} G_{i j} m_{i}+G_{i j}^{2} m_{j}-G_{i i} n_{j}\right) \\
\gamma_{i j} & =\frac{m_{i} m_{j}}{S_{i}} G_{i j}\left(G_{i i} m_{i}+G_{i j} m_{j}-n_{i}\right)
\end{aligned}
$$

with

$$
S_{i}=\left(G_{i i} m_{i}+G_{i j} m_{j}-n_{i}\right)\left(G_{i j} m_{i} n_{i}+G_{i j} m_{j} n_{j}-n_{i} n_{j}\right) .
$$

Here the symmetric matrix $G_{i j}$ is defined by Eq. (99). In Eqs. (65)-(67) the indices $i$ and $j$ belong to different particle species, $i \neq j$. For instance, if $i=1$ then $j=2$ and vice versa.

Now, let us return to Eqs. (61) and (62). The quantities $V_{\boldsymbol{k} \sigma}^{(i)}$ and $A_{\boldsymbol{k} \sigma}^{(i)}$, entering the kinetic equations (33)-(36), depend on the effective potentials $V_{\text {eff }}^{(i)}$ and $A_{\text {eff }}^{(i)}$ in exactly the same way as in the absence of Landau quasiparticle interaction [when $f^{i j}\left(\boldsymbol{k}, \boldsymbol{k}^{\prime}\right)=0$ ]. The only difference is that in all equations one should replace the bare mass $m_{i}$ with the effective mass $m_{i}^{*}$, or, equivalently, replace $m_{i}$ with $p_{\mathrm{F} i} / v_{\mathrm{F} i}$. Moreover, as follows from Eqs. (63) and (64), the gauge transformation properties of $V_{\text {eff }}^{(i)}$ and $\boldsymbol{A}_{\text {eff }}^{(i)}$ coincide with that of, respectively, $V$ and $\boldsymbol{A}$ [see Eqs. (50) and (51)]. Consequently, the relation between $\delta n_{i}, \boldsymbol{j}_{i}$ and $V_{\mathrm{eff}}^{(i)}, \boldsymbol{A}_{\mathrm{eff}}^{(i)}$ in an arbitrary gauge is given by the same expressions as for a noninteracting Fermi-liquid [compare with Eqs. (59) and (60)],

$$
\begin{aligned}
\delta n_{i} & =\Pi_{00}^{(i)}\left(V_{\mathrm{eff}}^{(i)}-\frac{\omega}{q} A_{\mathrm{eff}, \mathrm{l}}^{(i)}\right) \\
\boldsymbol{j}_{i} & =\Pi_{\mathrm{tr}}^{(i)} \boldsymbol{A}_{\mathrm{eff}, \mathrm{tr}}^{(i)}+\frac{\boldsymbol{q}}{\omega} \Pi_{\mathrm{l}}^{(i)}\left(V_{\mathrm{eff}}^{(i)}-\frac{\omega}{q} A_{\mathrm{eff}, \mathrm{l}}^{(i)}\right) .
\end{aligned}
$$


Let us emphasize once again that here the quantities $\Pi_{00}^{(i)}, \Pi_{1}^{(i)}$, and $\Pi_{\mathrm{tr}}^{(i)}$ should be understood as the functions of $p_{\mathrm{F} i}$ and $m_{i}^{*}$ (or $p_{\mathrm{F} i}$ and $v_{\mathrm{F} i}=p_{\mathrm{F} i} / m_{i}^{*}$ ) rather than the functions of $p_{\mathrm{F} i}$ and $m_{i}$. These two sets of variables are equivalent only for noninteracting Fermi-liquid, when $m_{i}^{*}=m_{i}$.

Now we are ready to find the polarization functions $P_{00}^{(i)}, P_{1}^{(i)}$, and $P_{\mathrm{tr}}^{(i)}$ for a two-component, strongly interacting Fermi-liquid. For that, we compare the general expressions (55) and (56) for $\delta n_{i}$ and $\boldsymbol{j}_{i}$ with the corresponding equations (68) and (69). Taking into account, that $V_{\mathrm{eff}}^{(i)}$ and $A_{\mathrm{eff}}^{(i)}$ are given by Eqs. (63) and (64), we obtain

$$
\begin{aligned}
P_{00}^{(i)} & =\frac{\Pi_{00}^{(i)}\left(e_{i} e_{j}+e_{j} \chi_{i j} \Pi_{00}^{(j)}-e_{i} \chi_{j j} \Pi_{00}^{(j)}\right)}{e_{i} e_{j}-e_{i} \chi_{j j} \Pi_{00}^{(j)}-e_{j} \chi_{i i} \Pi_{00}^{(i)}-\chi_{i j} \chi_{j i} \Pi_{00}^{(i)} \Pi_{00}^{(j)}+\chi_{i i} \chi_{j j} \Pi_{00}^{(i)} \Pi_{00}^{(j)}}, \\
P_{\mathrm{tr}}^{(i)} & =\frac{\Pi_{\mathrm{tr}}^{(i)}\left(e_{i} e_{j}+e_{j} \gamma_{i j} \Pi_{\mathrm{tr}}^{(j)}-e_{i} \gamma_{j j} \Pi_{\mathrm{tr}}^{(j)}\right)}{e_{i} e_{j}-e_{i} \gamma_{j j} \Pi_{\mathrm{tr}}^{(j)}-e_{j} \gamma_{i i} \Pi_{\mathrm{tr}}^{(i)}-\gamma_{i j} \gamma_{j i} \Pi_{\mathrm{tr}}^{(i)} \Pi_{\mathrm{tr}}^{(j)}+\gamma_{i i} \gamma_{j j} \Pi_{\mathrm{tr}}^{(i)} \Pi_{\mathrm{tr}}^{(j)}},
\end{aligned}
$$

while $P_{1}^{(i)}$ can be found from Eq. (54). Here $\chi_{i j}=f_{0}^{i j}-\left(\omega^{2} / q^{2}\right) \gamma_{i j}$ and the indices $i$ and $j$ refer to different particle species, $i \neq j$. Eqs. (70) and (71) will be analyzed in a subsequent section.

Here it is convenient to make a few comments, concerning the scheme of calculation of the polarization functions, suggested above. First, in principle it would be possible to extend this scheme taking into account the Landau parameters $f_{l}^{i j}$ with $l \geq 2$. However, for that one needs to know how the 'noninteracting' system $\left[\right.$ with $f^{i j}\left(\boldsymbol{k}, \boldsymbol{k}^{\prime}\right)=0$ ] responds to a general perturbation (not just to the electromagnetic field). This is a complex problem without any simple solution, such as in the case of $l<2$.

Second, the approach described above can be easily generalized to calculate the axial-vector response of the system. For that one needs to introduce the spin-dependent part of the Landau quasiparticle interaction in the kinetic equation (19). For a one-component superfluid Fermi-liquid, the axial-vector polarization functions were calculated by Leinson [9] under the simplified assumption that only nonzero Landau parameters are $g_{0}$ and $g_{1}$ (see Ref. [9] for the definition of $g_{0}$ and $\left.g_{1}\right)$. The generalization of his results to the case of mixtures is straightforward. The more difficult problem would be to estimate the effect of tensor quasinucleon interactions on the axial-vector response of the system. We hope to address this problem in our subsequent publication.

\section{Various limiting cases for $P_{00}^{(i)}$ and $P_{\mathrm{tr}}^{(i)}$}

We consider first a one-component Fermi-liquid. The particle species indices can then be suppressed. The polarization function $P_{00}$ for a one-component Fermi-liquid was studied by Leggett [23] in the limit of $q v_{\mathrm{F}} \ll \Delta$ and $\omega \ll \Delta$. Both polarization functions $P_{00}$ and $P_{\mathrm{tr}}$ were studied in the recent paper by Leinson [9] at arbitrary $q v_{\mathrm{F}} \ll \mu$ and $\omega \ll \mu$. In his analysis, Leinson took into account only the harmonic $V_{0}$ of the pairing interaction and assumed that $V_{l}=0$ for $l>0$. Our results will be compared with the results of these two authors.

For a one-component Fermi-liquid Eqs. (70) and (71) are essentially simplified

$$
\begin{aligned}
& P_{00}=\frac{e \Pi_{00}}{e-\chi \Pi_{00}}, \\
& P_{\mathrm{tr}}=\frac{e \Pi_{\mathrm{tr}}}{e-\gamma \Pi_{\mathrm{tr}}}
\end{aligned}
$$

with

$$
\begin{aligned}
& \gamma=-\frac{m m^{*}}{k_{\mathrm{F}}^{2}} f_{1}, \\
& \chi=f_{0}-\frac{\omega^{2}}{q^{2}} \gamma .
\end{aligned}
$$

To obtain Eqs. (72) and (73) we put $f_{0}^{i j}=0$ and $f_{1}^{i j}=0$ for $i \neq j$ in Eqs. (70) and (71) and then suppress the particle species indices.

Eq. (72) coincides with Eq. (68) of Leggett [23] and with Eq. (63) of Leinson [9] in the limit of $q v_{\mathrm{F}} \ll \Delta$ and $\omega \ll \Delta$. In the other limiting case, when $q v_{\mathrm{F}} \ll \Delta$ and $\omega>2 \Delta$, we reproduce the result of Leinson [9], see his Eq. (82). However, at arbitrary $q v_{\mathrm{F}}$ and $\omega$ our Eq. (72) differs from the general equation (55) for $P_{00}$, suggested by Leinson $[9]$. 
His Eq. (55) depends on two complicated combinations of integrals, $Q$ and $P$, that can be easily expressed through each other only in the limits discussed above. In contrast, our Eq. (72) depends solely on the polarization function $\Pi_{00}\left(p_{\mathrm{F}}, v_{\mathrm{F}}\right)$ of noninteracting one-component Fermi-liquid, which is the direct consequence of gauge invariance of the quantities $\delta n$ and $\boldsymbol{j}$.

Now let us examine Eq. (73) for $P_{\mathrm{tr}}$. Knowledge of $P_{\mathrm{tr}}$ allows one to calculate the transverse-current autocorrelation function $K_{T}$,

$$
K_{T}=\frac{P_{\mathrm{tr}}}{e}+\frac{n}{m} .
$$

This quantity was derived by Leinson (see Eq. (86) of Ref. [9]). Notice that his Eq. (86) contains a misprint [53]; one should replace $V_{F}$ with $p_{\mathrm{F}} / m$ in this equation. After correcting the misprint, Eq. (86) of Leinson coincides with our Eq. (76).

To further check Eq. (73), we look at the static limit of $P_{\mathrm{tr}}$, assuming that $\omega=0$ and $q v_{\mathrm{F}} \ll \Delta$. In the static limit the particle current density $\boldsymbol{j}$ is generated solely by a motion of superfluid liquid component (i.e., the normal component is at rest). It is given by [47]

$$
\boldsymbol{j}=\frac{\rho_{\mathrm{s}}}{m} \boldsymbol{V}_{\mathrm{s}},
$$

where $\rho_{\mathrm{s}}$ is the superfluid density and $\boldsymbol{V}_{\mathrm{s}}$ is the superfluid velocity. The velocity $\boldsymbol{V}_{\mathrm{s}}$ depends on the gauge-invariant combination of the phase $\varphi$ of the Cooper-pair condensate wave function and on the electromagnetic potential $\boldsymbol{A}$ (see, e.g., Ref. [47] and Sec. IV for more details),

$$
V_{\mathrm{s}}=\frac{1}{2 m}(\nabla \varphi-2 e \boldsymbol{A})
$$

In the transverse gauge in which $A_{1}=(\boldsymbol{q} \boldsymbol{A}) / q=0$, the phase $\varphi=0$, because it can only depend on the scalar $(\boldsymbol{q} A)=0$. Then it follows from Eqs. (77) and (78) that

$$
j=-\frac{e \rho_{\mathrm{s}}}{m^{2}} \boldsymbol{A}_{\mathrm{tr}} .
$$

For a one-component noninteracting Fermi-liquid

$$
\rho_{\mathrm{s}}=m n[1-\Phi(T)],
$$

where $\Phi(T)$ is a function of temperature [for more details see, e.g., Ref. 44], where this function was denoted by $f(T)$ ]. Using Eqs. (60), (79), and (80), one obtains

$$
\Pi_{\mathrm{tr}}\left(p_{\mathrm{F}}, v_{\mathrm{F}}\right)=-\frac{e n v_{\mathrm{F}}(1-\Phi)}{p_{\mathrm{F}}} .
$$

This equation together with Eqs. (7) and (73) gives

$$
P_{\mathrm{tr}}=-\frac{e n(1-\Phi)}{m\left(1+F_{1} \Phi / 3\right)}
$$

where $F_{1}=\left(m^{*} p_{\mathrm{F}} / \pi^{2}\right) f_{1}$. Comparing Eqs. (79) and (82), one can determine the superfluid density $\rho_{\mathrm{s}}$ for interacting Fermi-liquid,

$$
\rho_{\mathrm{s}}=-\frac{m^{2}}{e} P_{\mathrm{tr}}=\frac{m n[1-\Phi(T)]}{1+F_{1} \Phi(T) / 3}
$$

It coincides with the corresponding Eq. (72) of Leggett [44]. (Notice that, Leggett calculated the 'normal' density $\rho_{\mathrm{n}} \equiv m n-\rho_{\mathrm{s}}$.) Thus, we demonstrate that in the static limit our Eq. (73) reproduces the well-known result of Ref. [44].

We now turn our attention to the two-component Fermi-liquid and discuss first the static limit $\left(\omega=0\right.$ and $q v_{\mathrm{F} i} \ll$ $\Delta_{j}$ ). To obtain $P_{\mathrm{tr}}^{(i)}$ in this limit one should use Eq. (71) with $\Pi_{\mathrm{tr}}^{(i)}$ given by expression, similar to Eq. (81),

$$
\Pi_{\mathrm{tr}}^{(i)}\left(p_{\mathrm{F} i}, v_{\mathrm{F} i}\right)=-\frac{e_{i} n_{i} v_{\mathrm{F} i}\left(1-\Phi_{i}\right)}{p_{\mathrm{F} i}},
$$


where $\Phi_{i}$ is the same function of temperature as $\Phi$ (for more details, see Ref. [38]).

On the other hand, in the static limit the general hydrodynamics of superfluid mixtures is applicable, which states that in the absence of normal current (see, e.g., Ref. [36]),

$$
\boldsymbol{j}_{i}=\sum_{j} \frac{\rho_{i j}}{m_{i}} V_{\mathrm{s} j} .
$$

Here $\rho_{i j}$ is the entrainment matrix (also termed the Andreev-Bashkin or mass-density matrix) and $\boldsymbol{V}_{\mathrm{s} i}$ is the superfluid velocity for $i$-th particle species. It can be expressed through the phase $\varphi_{i}$ by an equation, similar to Eq. (78),

$$
\boldsymbol{V}_{\mathrm{si}}=\frac{1}{2 m_{i}}\left(\boldsymbol{\nabla} \varphi_{i}-2 e_{i} \boldsymbol{A}\right) .
$$

Again, as for a one-component liquid, in the transverse gauge $\varphi_{i}=0$ and from Eqs. (85) and (86) we have

$$
\boldsymbol{j}_{i}=-\left(\frac{e_{i} \rho_{i i}}{m_{i}^{2}}+\frac{e_{j} \rho_{i j}}{m_{i} m_{j}}\right) \boldsymbol{A}_{\mathrm{tr}}
$$

or, in view of Eq. (56),

$$
P_{\mathrm{tr}}^{(i)}=-\left(\frac{e_{i} \rho_{i i}}{m_{i}^{2}}+\frac{e_{j} \rho_{i j}}{m_{i} m_{j}}\right) .
$$

In Eqs. (87) and (88) indices $i$ and $j$ refer to different particle species, $i \neq j$. Comparing Eq. (88) with Eq. (171) and taking into account Eq. (84), one can determine the entrainment matrix $\rho_{i j}$ and verify that it coincides with the result of Ref. [38], obtained in a quite different way. (It should be noted that in Ref. [38] it is additionally demonstrated that the higher harmonics $f_{l}^{i j}$ with $l \geq 2$ do not contribute to $\rho_{i j}$.)

The next interesting limiting case is realized if one particle species, say, $i=1$, is charged while the other is not $\left(e_{2}=0\right)$. It follows then from general equation (70) that

$$
\begin{aligned}
P_{00}^{(1)} & =\frac{e_{1} \widetilde{\Pi}_{00}^{(1)}\left(1-\chi_{22} \widetilde{\Pi}_{00}^{(2)}\right)}{1-\chi_{22} \widetilde{\Pi}_{00}^{(2)}-\chi_{11} \widetilde{\Pi}_{00}^{(1)}-\chi_{12} \chi_{21} \widetilde{\Pi}_{00}^{(1)} \widetilde{\Pi}_{00}^{(2)}+\chi_{11} \chi_{22} \widetilde{\Pi}_{00}^{(1)} \widetilde{\Pi}_{00}^{(2)}}, \\
P_{00}^{(2)} & =\frac{e_{1} \chi_{21} \widetilde{\Pi}_{00}^{(1)} \widetilde{\Pi}_{00}^{(2)}}{1-\chi_{22} \widetilde{\Pi}_{00}^{(2)}-\chi_{11} \widetilde{\Pi}_{00}^{(1)}-\chi_{12} \chi_{21} \widetilde{\Pi}_{00}^{(1)} \widetilde{\Pi}_{00}^{(2)}+\chi_{11} \chi_{22} \widetilde{\Pi}_{00}^{(1)} \widetilde{\Pi}_{00}^{(2)}} .
\end{aligned}
$$

Here the function $\widetilde{\Pi}_{00}^{(i)}$ is independent of the electric charge $e_{i}, \widetilde{\Pi}_{00}^{(i)} \equiv \Pi_{00}^{(i)} / e_{i}$. An analogous expressions for $P_{\mathrm{tr}}^{(1)}$ and $P_{\mathrm{tr}}^{(2)}$ can be obtained with the help of Eq. (71). One sees from Eqs. (89) and (90) [and from the corresponding equations for $P_{\mathrm{tr}}^{(i)}$ ] that neutral particles not only modify the polarization function $P_{00 \text {, }}^{(1)}$ of charged particles, but also respond themselves to electromagnetic field, because $P_{00, t r}^{(2)} \neq 0$. However, the neutral particles do not contribute to the dielectric functions of the liquid, since, as follows from Eqs. (57) and (58), $\varepsilon_{1}$ and $\varepsilon_{\operatorname{tr}}$ are given by

$$
\begin{aligned}
\varepsilon_{1} & =1-\frac{4 \pi}{q^{2}} e_{1} P_{00}^{(1)}, \\
\varepsilon_{\mathrm{tr}} & =1-\frac{4 \pi}{\omega^{2}} e_{1} P_{\mathrm{tr}}^{(1)} .
\end{aligned}
$$

Finally, we mention another interesting property of the general solution (70) and (71). Assume that the polarization functions $\Pi_{00}^{(i=1,2)}\left[\right.$ or $\left.\Pi_{\mathrm{tr}}^{(i=1,2)}\right]$ of noninteracting Fermi-liquid are small so that one can neglect the terms of the order of $\Pi_{00}^{(i)} \Pi_{00}^{(j)}\left[\right.$ or $\Pi_{\mathrm{tr}}^{(i)} \Pi_{\mathrm{tr}}^{(j)}$. Then, as follows from Eqs. (70) and (71), in the linear approximation,

$$
P_{00}^{(i)} \approx \Pi_{00}^{(i)} \quad\left[\text { or } P_{\mathrm{tr}}^{(i)} \approx \Pi_{\mathrm{tr}}^{(i)}\right] .
$$

That is, the polarization functions are not modified by the first two harmonics $f_{0}^{i j}$ and $f_{1}^{i j}$ of the Landau quasiparticle interaction. In the next section we demonstrate that in some cases this conclusion is correct even if we take into account all harmonics $f_{l}^{i j}$ with $l \geq 0$. 


\section{Transverse polarization function in the Pippard limit}

In the Pippard limit we have $q v_{\mathrm{F} j} \gg \Delta_{i}, q v_{\mathrm{F} j} \gg \omega$, and $q v_{\mathrm{F} j} \ll \mu_{i}$. It is especially important to know the polarization functions $P_{00}^{(i)}$ and $P_{\mathrm{tr}}^{(i)}$ in this limit because they are required, for instance, for calculating the kinetic coefficients of a multi-fluid Fermi-mixture in neutron-star cores [33, 34].

In the first approximation, $P_{00}^{(i)}$ does not depend on the gap $\Delta_{i}$ and the frequency $\omega$ and agrees with the corresponding expression for normal matter, describing the ordinary static screening of particles. It can be easily obtained from Eq. (70) if we notice that for a normal one-component noninteracting Fermi-liquid one has (see, e.g., Ref. [45])

$$
\Pi_{00}^{(i)}=-\frac{e_{i} p_{\mathrm{F} i}^{2}}{\pi^{2} v_{\mathrm{F} i}}
$$

Strictly speaking, Eq. (70) that we employ, was derived under the assumption that the only harmonics $f_{0}^{i j}$ and $f_{1}^{i j}$ of the Landau quasiparticle interaction are nonzero. However, one can easily verify that, for a normal Fermi-liquid, $P_{00}^{(i)}$ is still given (at small $\boldsymbol{q}$ and $\omega$ ) by Eq. (70) even if we allow for higher harmonics $f_{l}^{i j}$ with $l \geq 2$. For a one-component Fermi-liquid this was demonstrated, for example, in Ref. [45].

Now let us consider the transverse polarization function $P_{\mathrm{tr}}^{(i)}$. In the Pippard limit the polarization function $\Pi_{\mathrm{tr}}^{(i)}$ is small, $\Pi_{\mathrm{tr}}^{(i)}=O\left[\Delta_{i} /\left(q v_{\mathrm{F} i}\right)+\omega /\left(q v_{\mathrm{F} i}\right)\right]$. It follows then, from the discussion at the end of the preceding section, that the first two harmonics $f_{0}^{i j}$ and $f_{1}^{i j}$ of the Landau quasiparticle interaction have no influence on $P_{\mathrm{tr}}^{(i)}$, so that it is given by Eq. (93).

Below we demonstrate that this result remains correct even if we take into account other harmonics $f_{l}^{i j}$ with $l \geq 2$. In the static limit $(\omega=0)$ this was first shown by Leggett [44]. More precisely, we prove that the transverse polarization function for a noninteracting system coincides with the function for a system with an arbitrary harmonic $f_{l}^{i j}(l \geq 2)$ switched on.

We consider first the simplified situation in which the pairing potential $V^{(i)}\left(\boldsymbol{k}, \boldsymbol{k}^{\prime}\right)$ is a constant; the generalization of our results to the case of an arbitrary $V^{(i)}\left(\boldsymbol{k}, \boldsymbol{k}^{\prime}\right)$ is briefly discussed at the end of the present section. In other words, we take into account only the first term $V_{0}^{(i)}$ in the expansion (12) of $V^{(i)}\left(\boldsymbol{k}, \boldsymbol{k}^{\prime}\right)$ in Legendre polynomials and neglect all other terms, $V_{l}^{(i)}=0$ for $l \geq 1$. In that case the functions $O_{\boldsymbol{k} \sigma+}^{(i)}$ and $O_{\boldsymbol{k} \sigma-}^{(i)}$ in Eqs. (33)-(36) are some constants depending on the scalars $\boldsymbol{q} \boldsymbol{A}$ and $V$ (see, e.g., Ref. [47]). In the transverse gauge, where $\boldsymbol{q} \boldsymbol{A}=\boldsymbol{q} \boldsymbol{A}_{\mathrm{tr}}=0$, and in the absence of scalar electromagnetic potential $V$, they vanish, $O_{\boldsymbol{k} \sigma+}^{(i)}=O_{\boldsymbol{k} \sigma-}^{(i)}=0$. The solution to the system of equations (33)-(36) is then simplified and we have for $\delta n_{\boldsymbol{k} \sigma+}^{(i)}\left(\boldsymbol{q}, \omega, \Delta_{i}\right)$ and $\delta n_{\boldsymbol{k} \sigma-}^{(i)}\left(\boldsymbol{q}, \omega, \Delta_{i}\right)$

$$
\begin{aligned}
& \delta n_{\boldsymbol{k} \sigma+}^{(i)}\left(\boldsymbol{q}, \omega, \Delta_{i}\right)=\left[\boldsymbol{q} \boldsymbol{v}_{i} A_{\boldsymbol{k} \sigma}^{(i)}-\omega V_{\boldsymbol{k} \sigma}^{(i)}\right] \frac{M_{1}}{D}, \\
& \delta n_{\boldsymbol{k} \sigma-}^{(i)}\left(\boldsymbol{q}, \omega, \Delta_{i}\right)=\omega A_{\boldsymbol{k} \sigma}^{(i)} \frac{M_{1}}{D}+V_{\boldsymbol{k} \sigma}^{(i)} \frac{M_{2}}{D},
\end{aligned}
$$

where

$$
\begin{aligned}
M_{1} & =\frac{1}{2} \boldsymbol{q} \boldsymbol{v}_{i}\left[4 \xi_{\boldsymbol{k}}^{(i) 2}-\omega^{2}\right]\left[F_{\boldsymbol{k}_{+}}^{(i)}+F_{\boldsymbol{k}_{-}}^{(i)}\right] \\
& +\xi_{\boldsymbol{k}}^{(i)}\left[4 \xi_{\boldsymbol{k}}^{(i) 2}+4 \Delta_{i}^{2}-\omega^{2}\right]\left[F_{\boldsymbol{k}_{+}}^{(i)}-F_{\boldsymbol{k}_{-}}^{(i)}\right] \\
M_{2} & =\frac{1}{2}\left[4 \Delta_{i}^{2} \omega^{2}-4\left(\boldsymbol{q} \boldsymbol{v}_{i}\right)^{2} \xi_{\boldsymbol{k}}^{(i) 2}+\left(\boldsymbol{q} \boldsymbol{v}_{i}\right)^{2} \omega^{2}\right]\left[F_{\boldsymbol{k}_{+}}^{(i)}+F_{\boldsymbol{k}_{-}}^{(i)}\right] \\
& +\boldsymbol{q} \boldsymbol{v}_{i} \xi_{\boldsymbol{k}}^{(i)}\left[4 \xi_{\boldsymbol{k}}^{(i) 2}-\omega^{2}\right]\left[F_{\boldsymbol{k}_{-}}^{(i)}-F_{\boldsymbol{k}_{+}}^{(i)}\right] \\
D & =\left(E_{\boldsymbol{k}_{-}}^{(i)}-E_{\boldsymbol{k}_{+}}^{(i)}-\omega-i 0\right)\left(E_{\boldsymbol{k}_{-}}^{(i)}+E_{\boldsymbol{k}_{+}}^{(i)}-\omega-i 0\right) \\
& \times\left(E_{\boldsymbol{k}_{-}}^{(i)}-E_{\boldsymbol{k}_{+}}^{(i)}+\omega+i 0\right)\left(E_{\boldsymbol{k}_{-}}^{(i)}+E_{\boldsymbol{k}_{+}}^{(i)}+\omega+i 0\right) .
\end{aligned}
$$

In Eqs. (95) and (96) $V_{\boldsymbol{k} \sigma}^{(i)}$ and $A_{\boldsymbol{k} \sigma}^{(i)}$ are the smooth functions of $\boldsymbol{k}$, defined in Eqs. (39) and (40), respectively. For our 
problem they can be rewritten as

$$
\begin{aligned}
V_{\boldsymbol{k} \sigma}^{(i)} & =\sum_{\boldsymbol{k}^{\prime} \sigma^{\prime} j} f_{l}^{i j} P_{l}(\cos \theta) \delta n_{\boldsymbol{k}^{\prime} \sigma^{\prime}-}^{(j)}\left(\boldsymbol{q}, \omega, \Delta_{j}\right) \\
A_{\boldsymbol{k} \sigma}^{(i)} & =2 \alpha_{i} \frac{\boldsymbol{k} \boldsymbol{A}}{m_{i}}-\sum_{\boldsymbol{k}^{\prime} \sigma^{\prime} j} f_{l}^{i j} P_{l}(\cos \theta) \delta n_{\boldsymbol{k}^{\prime} \sigma^{\prime}+}^{(j)}\left(\boldsymbol{q}, \omega, \Delta_{j}\right),
\end{aligned}
$$

where $\theta$ is the angle between $\boldsymbol{k}$ and $\boldsymbol{k}^{\prime}$ and $l \geq 2$.

As already mentioned above, Leggett [44] showed that the static function $P_{\mathrm{tr}}^{(i)}\left(\boldsymbol{q}, 0, \Delta_{i}\right)$ is not affected by the Landau quasiparticle interaction. Therefore, it is sufficient to analyze the difference $P_{\operatorname{tr}}^{(i)}\left(\boldsymbol{q}, \omega, \Delta_{i}\right)-P_{\mathrm{tr}}^{(i)}\left(\boldsymbol{q}, 0, \Delta_{i}\right)$ and prove that it is independent of $f_{l}^{i j}$. Using Eq. (46), we obtain

$$
\begin{aligned}
\boldsymbol{j}_{i}\left(\boldsymbol{q}, \omega, \Delta_{i}\right)-\boldsymbol{j}_{i}\left(\boldsymbol{q}, 0, \Delta_{i}\right) & =\left[P_{\mathrm{tr}}^{(i)}\left(\boldsymbol{q}, \omega, \Delta_{i}\right)-P_{\mathrm{tr}}^{(i)}\left(\boldsymbol{q}, 0, \Delta_{i}\right)\right] \boldsymbol{A}_{\mathrm{tr}} \\
& =\frac{1}{2} \sum_{\boldsymbol{k} \sigma} \frac{k}{m_{i}}\left[\delta n_{\boldsymbol{k} \sigma+}^{(i)}\left(\boldsymbol{q}, \omega, \Delta_{i}\right)-\delta n_{\boldsymbol{k} \sigma+}^{(i)}\left(\boldsymbol{q}, 0, \Delta_{i}\right)\right] .
\end{aligned}
$$

The function $\delta n_{\boldsymbol{k} \sigma+}^{(i)}\left(\boldsymbol{q}, \omega, \Delta_{i}\right)-\delta n_{\boldsymbol{k} \sigma+}^{(i)}\left(\boldsymbol{q}, 0, \Delta_{i}\right)$ is nonzero only in a narrow region near the Fermi surface, when $k \sim k_{\mathrm{F} i}$. Furthermore, because of the denominator $D$ [see Eq. (99)], this function has a sharp maximum at $\boldsymbol{q} \boldsymbol{v}_{i} \lesssim\left(\omega+\Delta_{j}\right)$. Introducing the longitudinal $\boldsymbol{k}_{1} \| \boldsymbol{q}$ and transverse $\boldsymbol{k}_{\mathrm{tr}} \perp \boldsymbol{q}$ components of the vector $\boldsymbol{k}=\boldsymbol{k}_{\mathrm{l}}+\boldsymbol{k}_{\mathrm{tr}}$, this inequality can be rewritten as

$$
k_{\mathrm{l}} \lesssim \frac{m_{i}\left(\omega+\Delta_{i}\right)}{q} \ll k_{\mathrm{tr}} \sim k_{\mathrm{F} i}
$$

The main contribution to the integral (102) comes from $k_{1}$ satisfying Eq. (103). Keeping this in mind, it is straightforward to verify (see, e.g., Ref. [51]) that for noninteracting Fermi-liquid one has

$$
\boldsymbol{j}_{i}\left(\boldsymbol{q}, \omega, \Delta_{i}\right)-\boldsymbol{j}_{i}\left(\boldsymbol{q}, 0, \Delta_{i}\right)=O\left(\frac{\omega+\Delta_{i}}{q v_{\mathrm{F} i}}\right) .
$$

Now let us analyze whether the Landau quasiparticle interaction influences this result. For this purpose we inspect the terms in the function $\delta n_{\boldsymbol{k} \sigma+}^{(i)}\left(\boldsymbol{q}, \omega, \Delta_{i}\right)-\delta n_{\boldsymbol{k} \sigma+}^{(i)}\left(\boldsymbol{q}, 0, \Delta_{i}\right)$ which depend on $f_{l}^{i j}$. From Eqs. (95), (100), and (101) it follows that they have the form,

$$
I=\sum_{\boldsymbol{k}^{\prime} \sigma^{\prime}} f_{l}^{i j} P_{l}\left(\frac{\boldsymbol{k} \boldsymbol{k}^{\prime}}{k_{\mathrm{F} i} k_{\mathrm{F} j}}\right) \delta n_{\boldsymbol{k}^{\prime} \sigma^{\prime}+}^{(j)} \quad \text { and } I I=\sum_{\boldsymbol{k}^{\prime} \sigma^{\prime}} f_{l}^{i j} P_{l}\left(\frac{\boldsymbol{k} \boldsymbol{k}^{\prime}}{k_{\mathrm{F} i} k_{\mathrm{F} j}}\right) \delta n_{\boldsymbol{k}^{\prime} \sigma^{\prime}-}^{(j)} .
$$

By demonstrating that these integrals are quadratic in $\left(\omega+\Delta_{j}\right) /\left(q v_{\mathrm{F} i}\right)$ we prove that $P_{\mathrm{tr}}^{(i)}=\Pi_{\mathrm{tr}}^{(i)}\left(p_{\mathrm{F} i}, v_{\mathrm{F} i}\right)$ in the Pippard limit. Below we consider in detail the first term in Eq. (105); the analysis of the second term is similar.

The first term can be presented as

$$
\begin{aligned}
I & =\sum_{\boldsymbol{k}^{\prime} \sigma^{\prime}} f_{l}^{i j} P_{l}\left(\frac{\boldsymbol{k} \boldsymbol{k}^{\prime}}{k_{\mathrm{F} i} k_{\mathrm{F} j}}\right)\left[\delta n_{\boldsymbol{k}^{\prime} \sigma^{\prime}+}^{(j)}\left(\boldsymbol{q}, \omega, \Delta_{j}\right)-\delta n_{\boldsymbol{k}^{\prime} \sigma^{\prime}+}^{(j)}\left(\boldsymbol{q}, 0, \Delta_{j}\right)\right] \\
& +\sum_{\boldsymbol{k}^{\prime} \sigma^{\prime}} f_{l}^{i j} P_{l}\left(\frac{\boldsymbol{k} \boldsymbol{k}^{\prime}}{k_{\mathrm{F} i} k_{\mathrm{F} j}}\right)\left[\delta n_{\boldsymbol{k}^{\prime} \sigma^{\prime}+}^{(j)}\left(\boldsymbol{q}, 0, \Delta_{j}\right)-\delta n_{\boldsymbol{k}^{\prime} \sigma^{\prime}+}^{(j)}(\boldsymbol{q}, 0,0)\right] \\
& +\sum_{\boldsymbol{k}^{\prime} \sigma^{\prime}} f_{l}^{i j} P_{l}\left(\frac{\boldsymbol{k} \boldsymbol{k}^{\prime}}{k_{\mathrm{F} i} k_{\mathrm{F} j}}\right) \delta n_{\boldsymbol{k}^{\prime} \sigma^{\prime}+}^{(j)}(\boldsymbol{q}, 0,0) .
\end{aligned}
$$

One can easily verify that the last integral here vanishes at $l \neq 1$ and thus can be omitted. Furthermore, because of the same reasons as those discussed below Eq. (102), the main contribution to the first two integrals in Eq. (106) comes from such a region of $\boldsymbol{k}^{\prime}$, that

$$
k_{\mathrm{l}}^{\prime} \lesssim \frac{m_{j}\left(\omega+\Delta_{j}\right)}{q} \ll k_{\mathrm{tr}}^{\prime} \sim k_{\mathrm{F} j}
$$


From symmetry arguments it follows that the functions in square brackets in these integrals can generally be written in the form

$$
[\ldots]=\left(\boldsymbol{k}^{\prime} \boldsymbol{A}\right) G\left(\boldsymbol{k}^{\prime} \boldsymbol{q}, k^{\prime}\right)
$$

where $G\left(\boldsymbol{k}^{\prime} \boldsymbol{q}, k^{\prime 2}\right)$ is a scalar function.

Now, using Eqs. (103), (107), and (108), one may write

$$
\begin{aligned}
& \sum_{\boldsymbol{k}^{\prime} \sigma^{\prime}} f_{l}^{i j} P_{l}\left(\frac{\boldsymbol{k}^{\prime}}{k_{\mathrm{F} i} k_{\mathrm{F} j}}\right)[\ldots]=\sum_{\boldsymbol{k}^{\prime} \sigma^{\prime}} f_{l}^{i j} P_{l}\left(\frac{k_{\mathrm{l}} k_{1}^{\prime}+\boldsymbol{k}_{\mathrm{tr}} \boldsymbol{k}_{\mathrm{tr}}^{\prime}}{k_{\mathrm{F} i} k_{\mathrm{F} j}}\right)\left(\boldsymbol{k}^{\prime} \boldsymbol{A}\right) G\left(\boldsymbol{k}^{\prime} \boldsymbol{q}, k^{\prime}\right) \\
= & \sum_{k_{1}^{\prime} \boldsymbol{k}_{\mathrm{tr}}^{\prime} \sigma^{\prime}} f_{l}^{i j} P_{l}\left(\frac{\boldsymbol{k}_{\mathrm{tr}} \boldsymbol{k}_{\mathrm{tr}}^{\prime}}{k_{\mathrm{F} i} k_{\mathrm{F} j}}\right)\left(\boldsymbol{k}_{\mathrm{tr}}^{\prime} A_{\mathrm{tr}}\right) G\left(k_{1}^{\prime} q, k^{\prime}\right)+O\left(\frac{\left(\omega+\Delta_{i}\right)}{q v_{\mathrm{F} i}} \frac{\left(\omega+\Delta_{j}\right)}{q v_{\mathrm{F} j}}\right) \\
= & O\left(\frac{\left(\omega+\Delta_{i}\right)}{q v_{\mathrm{F} i}} \frac{\left(\omega+\Delta_{j}\right)}{q v_{\mathrm{F} j}}\right) .
\end{aligned}
$$

Here we used the fact that the integral over directions of $\boldsymbol{k}_{\mathrm{tr}}^{\prime}$ vanishes for $l \neq 1$. Thus, we demonstrate that $I \sim$ $\left(\omega+\Delta_{i}\right) /\left(q v_{\mathrm{F} i}\right) \times\left(\omega+\Delta_{j}\right) /\left(q v_{\mathrm{F} j}\right)$ and, consequently, the Landau quasiparticle interaction has no influence on $P_{\mathrm{tr}}^{(i)}$ in the Pippard limit.

The consideration of this section is simplified since we take into account only the harmonic $V_{0}^{(i)}$ of the pairing potential. However, it seems plausible (though we have not checked it in detail) that the inclusion of other harmonics $V_{l}^{(i)}$ with $l \geq 1$ will not change the result. In principle, a prove of this more general statement should be similar to the prove presented here, but the equations to be analyzed, are much more complicated. In particular, the function $\delta n_{\boldsymbol{k} \sigma+}^{(i)}$ will depend, in addition, on the integrals $O_{\boldsymbol{k} \sigma+}^{(i)}$ and $O_{\boldsymbol{k} \sigma-}^{(i)}$ [see Eqs. (37) and (38)] .

Finally, let us make a comment concerning the coefficients $f_{1}^{i j}$ and $V_{1}^{(i)}$ of the first harmonic. From the analysis presented above it is clear that the coefficient $f_{1}^{i j}$ plays a very special role, because the integrals in Eqs. (106) and (109) do not vanish at $l=1$. It might think that the situation with the coefficient $V_{1}^{(i)}$ of the pairing potential is analogous, so that the integrals

$$
\sum_{\boldsymbol{k}^{\prime} \sigma} V_{1}^{(i)} P_{1}(\cos \theta) \delta s_{\boldsymbol{k}^{\prime} \sigma-}^{(i)}\left(\boldsymbol{q}, \omega, \Delta_{i}\right) \text { and } \sum_{\boldsymbol{k}^{\prime} \sigma} V_{1}^{(i)} P_{1}(\cos \theta) \delta s_{\boldsymbol{k}^{\prime} \sigma+}^{(i)}\left(\boldsymbol{q}, \omega, \Delta_{i}\right)
$$

are nonzero. However, this is not the case; they vanish due to the symmetry relations (31) and (32).

\section{A PHENOMENOLOGICAL APPROACH TO KINETIC EQUATION AT SMALL $q$ AND $\omega$}

In this section we analyze the kinetic equation in the 'quasiclassical' limit, $q v_{\mathrm{F} i} \ll \Delta_{j}$ and $\omega \ll \Delta_{j}$ (see, e.g., Refs. [24, 44]). This limit is especially important for various applications, for instance, to study low-frequency longwavelength collective modes propagating in superfluid matter and to calculate kinetic coefficients.

For a one-component Fermi-liquid the quasiclassical limit of the matrix kinetic equation (19) was thoroughly examined in Ref. [24]. It was demonstrated, that Eq. (19) can be substantially simplified by introducing a concept of Bogoliubov excitations. In particular, the kinetic equation for Bogoliubov excitations acquires a scalar (rather than matrix) form. For a two-component Fermi-mixture the analysis is quite similar. Here we do not attempt to perform it; an interested reader is referred to Ref. 24] for more details. Instead, we follow a more intuitive phenomenological approach allowing to formulate the kinetic equation in the non-linear regime (in contrast to Ref. 24], where the kinetic equation is studied only in the linear approximation). We call this regime non-linear in a sense that, for instance, it allows us to study the nonequilibrium variations of the energy gap which are comparable to $\Delta_{i}$. However, because we use the Landau theory of Fermi-liquids, we still assume that the quasiparticle distribution only slightly differs (in the vicinity of the Fermi surface) from the step function. Our results will be discussed and compared with those available in the literature in the end of the present section.

In the previous sections the quasiparticle momentum was denoted as $\boldsymbol{k}$. In the Appendix A we demonstrate that in the presence of electromagnetic field $\boldsymbol{k}$ is actually a generalized momentum. To distinguish between $\boldsymbol{k}$ and the real momentum, the latter will be denoted by $\boldsymbol{p}$. It is more convenient to use $\boldsymbol{p}$ instead of $\boldsymbol{k}$ in the consideration below. 


\section{A. Local analysis}

In the quasiclassical limit one can assume that a Landau quasiparticle (or a Bogoliubov excitation) with a certain momentum $\boldsymbol{p}$ possesses, at the same time, a certain coordinate $\boldsymbol{r}$. Consequently, such quantities as the distribution of Landau quasiparticles (Bogoliubov excitations), their energy, or the energy gap can be considered as functions of $\boldsymbol{p}$ and $\boldsymbol{r}$. To find how these quantities are related to each other it is sufficient to analyze the system locally.

Let us consider a two-component Fermi-liquid out of thermodynamic equilibrium. To simplify the problem we neglect for a while the electromagnetic field, assuming that the liquid is composed of neutral particles. The electromagnetic effects will be taken into account in Sec. IVC. Our aim will be to calculate the energy density $E$ of superfluid matter in the neighborhood of a point $\boldsymbol{r}$. In the vicinity of this point the matter is almost homogeneous. Thus, it can be approximately described by a uniform Hamiltonian $H$ (see, e.g., Refs. [38, 44, 49]),

$$
H-\sum_{i} \breve{\mu}_{i} N_{i}=H_{\mathrm{LF}}+H_{\text {pairing }}
$$

where $N_{i}$ is the number density operator; $\breve{\mu}_{i}$ is the nonequilibrium analogue of the chemical potential $\mu_{i}$ to be determined below; $H_{\mathrm{LF}}$ is the Fermi-liquid Hamiltonian,

$$
\begin{aligned}
H_{\mathrm{LF}} & =\sum_{\boldsymbol{p} \sigma i}\left(\varepsilon_{\boldsymbol{p} 0}^{(i)}-\breve{\mu}_{i}\right)\left(a_{\boldsymbol{p} \sigma}^{(i) \dagger} a_{\boldsymbol{p} \sigma}^{(i)}-\theta_{\boldsymbol{p}}^{(i)}\right) \\
& +\frac{1}{2} \sum_{\boldsymbol{p} \boldsymbol{p}^{\prime} \sigma \sigma^{\prime} i j} f^{i j}\left(\boldsymbol{p}, \boldsymbol{p}^{\prime}\right)\left(a_{\boldsymbol{p} \sigma}^{(i) \dagger} a_{\boldsymbol{p} \sigma}^{(i)}-\theta_{\boldsymbol{p}}^{(i)}\right)\left(a_{\boldsymbol{p}^{\prime} \sigma^{\prime}}^{(j) \dagger} a_{\boldsymbol{p}^{\prime} \sigma^{\prime}}^{(j)}-\theta_{\boldsymbol{p}^{\prime}}^{(j)}\right),
\end{aligned}
$$

and $H_{\text {pairing }}$ is the pairing Hamiltonian. In the presence of superfluid currents it is given by (see, e.g., Ref. [38])

$$
H_{\text {pairing }}=\sum_{\boldsymbol{p} \boldsymbol{p}^{\prime} i} V_{\boldsymbol{Q}_{i}}^{(i)}\left(\boldsymbol{p}, \boldsymbol{p}^{\prime}\right) a_{\boldsymbol{p}^{\prime}+\boldsymbol{Q}_{i} \uparrow}^{(i) \dagger} a_{-\boldsymbol{p}^{\prime}+\boldsymbol{Q}_{i} \downarrow}^{(i) \dagger} a_{-\boldsymbol{p}+\boldsymbol{Q}_{i} \downarrow}^{(i)} a_{\boldsymbol{p}+\boldsymbol{Q}_{i} \uparrow}^{(i)}
$$

Here, $2 \boldsymbol{Q}_{i}=2 m_{i} V_{\mathrm{s} i}$ is the momentum of a Cooper-pair in the condensate. It is related by Eqs. (143) and (145) to the quantity $O_{\boldsymbol{k} \sigma-}^{(i)}$, introduced in Sec. IIB. The matrix element $V_{\boldsymbol{Q}_{i}}^{(i)}\left(\boldsymbol{p}, \boldsymbol{p}^{\prime}\right)$ in Eq. (113) describes scattering of a pair of Landau quasiparticles from the states $\left(\boldsymbol{p}+\boldsymbol{Q}_{i}, \uparrow\right),\left(-\boldsymbol{p}+\boldsymbol{Q}_{i}, \downarrow\right)$ to states $\left(\boldsymbol{p}^{\prime}+\boldsymbol{Q}_{i}, \uparrow\right),\left(-\boldsymbol{p}^{\prime}+\boldsymbol{Q}_{i}, \downarrow\right)$. In Ref. 38] it is argued that $V_{\boldsymbol{Q}_{i}}^{(i)}\left(\boldsymbol{p}, \boldsymbol{p}^{\prime}\right) \approx V^{(i)}\left(\boldsymbol{p}, \boldsymbol{p}^{\prime}\right)$ [up to small terms $\left.\sim\left(Q_{i} / k_{\mathrm{Fj}}\right)^{2}\right]$.

To find the energy density $E$ one needs to diagonalize the Hamiltonian (111). To do that, we rewrite Eq. (111) in terms of Bogoliubov operators $b_{\boldsymbol{p} \sigma}^{(i)}$, defined as (see, e.g., Ref. [38])

$$
\begin{aligned}
& a_{\boldsymbol{p}+\boldsymbol{Q}_{i} \uparrow}^{(i)}=u_{\boldsymbol{p}}^{(i)} b_{\boldsymbol{p}+\boldsymbol{Q}_{i} \uparrow}^{(i)}+v_{\boldsymbol{p}}^{(i)} b_{-\boldsymbol{p}+\boldsymbol{Q}_{i} \downarrow}^{(i) \dagger}, \\
& a_{\boldsymbol{p}+\boldsymbol{Q}_{i} \downarrow}^{(i)}=u_{\boldsymbol{p}}^{(i)} b_{\boldsymbol{p}+\boldsymbol{Q}_{i} \downarrow}^{(i)}-v_{\boldsymbol{p}}^{(i)} b_{-\boldsymbol{p}+\boldsymbol{Q}_{i} \uparrow}^{(i) \dagger},
\end{aligned}
$$

where $u_{\boldsymbol{p}}^{(i)}$ and $v_{\boldsymbol{p}}^{(i)}$ are even functions of $\boldsymbol{p}$,

$$
u_{\boldsymbol{p}}^{(i)}=u_{-\boldsymbol{p}}^{(i)}, \quad v_{\boldsymbol{p}}^{(i)}=v_{-\boldsymbol{p}}^{(i)},
$$

normalized by the condition

$$
u_{\boldsymbol{p}}^{(i) 2}+v_{\boldsymbol{p}}^{(i) 2}=1
$$

Generally, the coefficients $u_{\boldsymbol{p}}^{(i)}$ and $v_{\boldsymbol{p}}^{(i)}$ are complex. However, at some moment of time they can be chosen to be real in the vicinity of our point $\boldsymbol{r}$ by a suitable phase transformation.

Being expressed through the Bogoliubov operators, the Hamiltonian (111) takes the diagonal form. Thus, one gets the following expression for the energy density

$$
\begin{gathered}
E-\sum_{i} \breve{\mu}_{i} n_{i}=\sum_{\boldsymbol{p} \sigma i}\left[\varepsilon_{\boldsymbol{p}+\boldsymbol{Q}_{i} 0}^{(i)}-\breve{\mu}_{i}\right]\left(\mathcal{N}_{\boldsymbol{p}+\boldsymbol{Q}_{i}}^{(i)}-\theta_{\boldsymbol{p}+\boldsymbol{Q}_{i}}^{(i)}\right) \\
+\frac{1}{2} \sum_{\boldsymbol{p} \boldsymbol{p}^{\prime} \sigma \sigma^{\prime} i j} f^{i j}\left(\boldsymbol{p}+\boldsymbol{Q}_{i}, \boldsymbol{p}^{\prime}+\boldsymbol{Q}_{j}\right)\left(\mathcal{N}_{\boldsymbol{p}+\boldsymbol{Q}_{i}}^{(i)}-\theta_{\boldsymbol{p}+\boldsymbol{Q}_{i}}^{(i)}\right)\left(\mathcal{N}_{\boldsymbol{p}^{\prime}+\boldsymbol{Q}_{j}}^{(j)}-\theta_{\boldsymbol{p}^{\prime}+\boldsymbol{Q}_{j}}^{(j)}\right) \\
+\sum_{\boldsymbol{p} \boldsymbol{p}^{\prime} i} V^{(i)}\left(\boldsymbol{p}, \boldsymbol{p}^{\prime}\right) u_{\boldsymbol{p}}^{(i)} v_{\boldsymbol{p}}^{(i)} u_{\boldsymbol{p}^{\prime}}^{(i)} v_{\boldsymbol{p}^{\prime}}^{(i)} \\
\times\left(1-\mathcal{F}_{\boldsymbol{p}+\boldsymbol{Q}_{i}}^{(i)}-\mathcal{F}_{-\boldsymbol{p}+\boldsymbol{Q}_{i}}^{(i)}\right)\left(1-\mathcal{F}_{\boldsymbol{p}^{\prime}+\boldsymbol{Q}_{i}}^{(i)}-\mathcal{F}_{-\boldsymbol{p}^{\prime}+\boldsymbol{Q}_{i}}^{(i)}\right) .
\end{gathered}
$$


Here $\mathcal{F}_{\boldsymbol{p}+\boldsymbol{Q}_{i}}^{(i)}$ is the distribution function for Bogoliubov excitations with momentum $\left(\boldsymbol{p}+\boldsymbol{Q}_{i}\right)$,

$$
\mathcal{F}_{\boldsymbol{p}+\boldsymbol{Q}_{i}}^{(i)}=\left\langle\left|b_{\boldsymbol{p}+\boldsymbol{Q}_{i} \uparrow}^{(i) \dagger} b_{\boldsymbol{p}+\boldsymbol{Q}_{i} \uparrow}^{(i)}\right|\right\rangle=\left\langle\left|b_{\boldsymbol{p}+\boldsymbol{Q}_{i} \downarrow}^{(i) \dagger} b_{\boldsymbol{p}+\boldsymbol{Q}_{i} \downarrow}^{(i)}\right|\right\rangle,
$$

while $\mathcal{N}_{\boldsymbol{p}+\boldsymbol{Q}_{i}}^{(i)}$ is the average number of Landau quasiparticles in a state $\left(\boldsymbol{p}+\boldsymbol{Q}_{i}, \sigma\right)$,

$$
\begin{aligned}
\mathcal{N}_{\boldsymbol{p}+\boldsymbol{Q}_{i}}^{(i)} & =\left\langle\left|a_{\boldsymbol{p}+\boldsymbol{Q}_{i} \uparrow}^{(i) \dagger} a_{\boldsymbol{p}+\boldsymbol{Q}_{i} \uparrow}^{(i)}\right|\right\rangle=\left\langle\left|a_{\boldsymbol{p}+\boldsymbol{Q}_{i} \downarrow}^{(i) \dagger} a_{\boldsymbol{p}+\boldsymbol{Q}_{i} \downarrow}^{(i)}\right|\right\rangle \\
& =v_{\boldsymbol{p}}^{(i) 2}+u_{\boldsymbol{p}}^{(i) 2} \mathcal{F}_{\boldsymbol{p}+\boldsymbol{Q}_{i}}^{(i)}-v_{\boldsymbol{p}}^{(i) 2} \mathcal{F}_{-\boldsymbol{p}+\boldsymbol{Q}_{i}}^{(i)}
\end{aligned}
$$

If we were in thermodynamic equilibrium we could easily find the unknown functions $\mathcal{F}_{\boldsymbol{p}+\boldsymbol{Q}_{\boldsymbol{i}}}^{(i)}$ and $u_{\boldsymbol{p}}^{(i)}$ in Eq. (118) by requiring minimum of the free energy $F, F\left[\mathcal{F}_{\boldsymbol{p}+\boldsymbol{Q}_{i}}^{(i)}, u_{\boldsymbol{p}}^{(i)}\right] \equiv E-\breve{\mu}_{1} n_{1}-\breve{\mu}_{2} n_{2}-T S$, where the entropy $S\left[\mathcal{F}_{\boldsymbol{p}+\boldsymbol{Q}_{i}}^{(i)}\right]$ is the functional of only $\mathcal{F}_{\boldsymbol{p}+\boldsymbol{Q}_{\boldsymbol{i}}}^{(i)}$ (see Ref. [38] for more details). Since we are not in thermodynamic equilibrium, the distribution function for Bogoliubov excitations $\mathcal{F}_{\boldsymbol{p}+\boldsymbol{Q}_{\boldsymbol{i}}}^{(i)}$ in our local analysis should be considered as a given 'input parameter' [it can be found from the corresponding Boltzmann kinetic equation (132), see Sec. IVB]. To determine $u_{\boldsymbol{p}}^{(i)}$, we assume that even out of equilibrium $F$ still has a minimum as the functional of $u_{\boldsymbol{p}}^{(i)}$ (at fixed $\mathcal{F}_{\boldsymbol{p}+\boldsymbol{Q}_{\boldsymbol{i}}}^{(i)}$ ). This assumption, though plausible, cannot be proven in our phenomenological approach. However, its validity can be justified by comparison with the results of the strict microscopic theory (see Sec. IVC).

One obtains from the minimization procedure

$$
u_{\boldsymbol{p}}^{(i) 2}=\frac{1}{2}\left(1+\frac{H_{\boldsymbol{p}+\boldsymbol{Q}_{i}}^{(i)}+H_{-\boldsymbol{p}+\boldsymbol{Q}_{i}}^{(i)}}{2 \mathfrak{E}_{\boldsymbol{p}+\boldsymbol{Q}_{i}}^{(i)}+H_{-\boldsymbol{p}+\boldsymbol{Q}_{i}}^{(i)}-H_{\boldsymbol{p}+\boldsymbol{Q}_{i}}^{(i)}}\right),
$$

where

$$
H_{\boldsymbol{p}}^{(i)}=\varepsilon_{\boldsymbol{p}}^{(i)}\left[\mathcal{N}_{\boldsymbol{p}}^{(j)}\right]-\breve{\mu}_{i}
$$

see Eq. (11) for the definition of $\varepsilon_{\boldsymbol{p}}^{(i)}$, and

$$
\mathfrak{E}_{\boldsymbol{p}+\boldsymbol{Q}_{i}}^{(i)}=\frac{1}{2}\left(H_{\boldsymbol{p}+\boldsymbol{Q}_{i}}^{(i)}-H_{-\boldsymbol{p}+\boldsymbol{Q}_{i}}^{(i)}\right)+\sqrt{\frac{1}{4}\left(H_{\boldsymbol{p}+\boldsymbol{Q}_{i}}^{(i)}+H_{-\boldsymbol{p}+\boldsymbol{Q}_{i}}^{(i)}\right)^{2}+\mathcal{D}_{\boldsymbol{p}}^{(i) 2}}
$$

is the energy of a Bogoliubov excitation with momentum $\left(\boldsymbol{p}+\boldsymbol{Q}_{i}\right)$. To verify that $\mathfrak{E}_{\boldsymbol{p}+\boldsymbol{Q}_{i}}^{(i)}$ is indeed the energy, it is sufficient to notice that it is given by the variational derivative of the functional $\left(E-\sum_{i} \breve{\mu}_{i} n_{i}\right)$ with respect to $\mathcal{F}_{\boldsymbol{p}+\boldsymbol{Q}_{i}}^{(i)}$,

$$
\mathfrak{E}_{\boldsymbol{p}+\boldsymbol{Q}_{i}}^{(i)}=\frac{\delta\left(E-\sum_{i} \breve{\mu}_{i} n_{i}\right)}{\delta \mathcal{F}_{\boldsymbol{p}+\boldsymbol{Q}_{i}}^{(i)}}
$$

Finally, $\mathcal{D}_{\boldsymbol{p}}^{(i)}$ in Eq. (123) is the nonequilibrium energy gap. It is defined by the equation,

$$
\mathcal{D}_{\boldsymbol{p}}^{(i)}=-\sum_{\boldsymbol{p}^{\prime}} V^{(i)}\left(\boldsymbol{p}, \boldsymbol{p}^{\prime}\right) u_{\boldsymbol{p}^{\prime}}^{(i)} v_{\boldsymbol{p}^{\prime}}^{(i)}\left(1-\mathcal{F}_{\boldsymbol{p}^{\prime}+\boldsymbol{Q}_{i}}^{(i)}-\mathcal{F}_{-\boldsymbol{p}^{\prime}+\boldsymbol{Q}_{i}}^{(i)}\right)
$$

It can be demonstrated that, in the linear approximation of Sec. II, the quantity $\delta \mathcal{D}_{\boldsymbol{p}}^{(i)} \equiv \mathcal{D}_{\boldsymbol{p}}^{(i)}-\Delta_{i}$ is related to the integral $O_{\boldsymbol{p} \sigma+}^{(i)}$ [see Eq. (37)],

$$
\delta \mathcal{D}_{\boldsymbol{p}}^{(i)}=\frac{O_{\boldsymbol{p} \sigma+}^{(i)}}{2}
$$

Using Eq. (120) one can determine the nonequilibrium chemical potential $\breve{\mu}_{i}$ from the requirement that the number density $n_{i}$ is given by the sum over all occupied quasiparticle states,

$$
n_{i}=\sum_{\boldsymbol{p} \sigma} \mathcal{N}_{\boldsymbol{p}+\boldsymbol{Q}_{i}}^{(i)}
$$


The quantities $\mathcal{N}_{\boldsymbol{p}+\boldsymbol{Q}_{i}}^{(i)}, \mathfrak{E}_{\boldsymbol{p}+\boldsymbol{Q}_{i}}^{(i)}$, and $\mathcal{D}_{\boldsymbol{p}}^{(i)}$ can be easily found from, respectively, Eqs. (120), (123), and (125), once the distribution $\mathcal{F}_{\boldsymbol{p}+\boldsymbol{Q}_{\boldsymbol{i}}}^{(i)}$ is specified. As shown in Ref. [38], in thermodynamic equilibrium the function $\mathcal{F}_{\boldsymbol{p}+\boldsymbol{Q}_{\boldsymbol{i}}}^{(i)}$ is given by the standard Fermi-Dirac distribution,

$$
\widetilde{\mathcal{F}}_{\boldsymbol{p}+\boldsymbol{Q}_{i} 0}^{(i)}=\frac{1}{1+\mathrm{e}^{\mathfrak{E}_{\boldsymbol{p}+\boldsymbol{Q}_{i}}^{(i)} / T}}
$$

Here and below the equilibrium function $\mathcal{F}_{\boldsymbol{p}}^{(i)}$ is denoted as $\widetilde{\mathcal{F}}_{\boldsymbol{p} 0}^{(i)}$, where tilde indicates that we allow for superfluid currents in the system. The function $\widetilde{\mathcal{F}}_{\boldsymbol{p} 0}^{(i)}$ should not be confused with the distribution $\mathcal{F}_{\boldsymbol{p} 0}^{(i)}$ [see Sec. IIA and, in particular, Eq. (5) for the definition of $\mathcal{F}_{p 0}^{(i)}$ ]. These functions are equal only in the absence of superfluid currents $\left(\boldsymbol{Q}_{i}=0\right)$,

$$
\widetilde{\mathcal{F}}_{\boldsymbol{p} 0}^{(i)}=\mathcal{F}_{\boldsymbol{p} 0}^{(i)}
$$

In this case one also has for the system in thermodynamic equilibrium

$$
\begin{gathered}
\mathfrak{E}_{\boldsymbol{p}}^{(i)}=E_{\boldsymbol{p}}^{(i)}, \\
\mathcal{D}_{\boldsymbol{p}}^{(i)}=\Delta_{i} .
\end{gathered}
$$

The equalities (129)-(131) follow from Eqs. (4), (5), and (10) of Sec. IIA and Eqs. (120)-(128) of the present section (see Ref. [38] for a detailed derivation).

\section{B. Introducing dynamics}

The relations between various nonequilibrium quantities discussed above should be supplemented by the kinetic equation for $\mathcal{F}_{\boldsymbol{p}+\boldsymbol{Q}_{i}}^{(i)}$, the continuity equation, and by the 'superfluid' equation, describing the evolution of $\boldsymbol{Q}_{i}$ with time $t$. The kinetic equation for the Bogoliubov excitations takes the standard form,

$$
\frac{\partial \mathcal{F}_{\boldsymbol{p}+\boldsymbol{Q}_{i}}^{(i)}}{\partial t}+\frac{\partial \mathfrak{E}_{\boldsymbol{p}+\boldsymbol{Q}_{i}}^{(i)}}{\partial \boldsymbol{p}} \frac{\partial \mathcal{F}_{\boldsymbol{p}+\boldsymbol{Q}_{i}}^{(i)}}{\partial \boldsymbol{r}}-\frac{\partial \mathfrak{E}_{\boldsymbol{p}+\boldsymbol{Q}_{i}}^{(i)}}{\partial \boldsymbol{r}} \frac{\partial \mathcal{F}_{\boldsymbol{p}+\boldsymbol{Q}_{i}}^{(i)}}{\partial \boldsymbol{p}}=\operatorname{St}\left\{\mathcal{F}_{\boldsymbol{p}+\boldsymbol{Q}_{j}}^{(j=1,2)}\right\} .
$$

The collision integral on the right-hand side of this equation can be easily obtained once the interaction between the excitations is known (see, e.g., Refs. [25 30, 54]).

The continuity equation is written as

$$
\frac{\partial n_{i}}{\partial t}+\operatorname{div} \boldsymbol{j}_{i}=0
$$

where the number density equals

$$
n_{i}=\sum_{\boldsymbol{p} \sigma} \mathcal{N}_{\boldsymbol{p}+\boldsymbol{Q}_{i}}=\sum_{\boldsymbol{p} \sigma}\left[v_{\boldsymbol{p}}^{(i) 2}+u_{\boldsymbol{p}}^{(i) 2} \mathcal{F}_{\boldsymbol{p}+\boldsymbol{Q}_{i}}^{(i)}-v_{\boldsymbol{p}}^{(i) 2} \mathcal{F}_{-\boldsymbol{p}+\boldsymbol{Q}_{i}}^{(i)}\right]
$$

and the particle current density is given by (see, e.g., Ref. [44])

$$
\boldsymbol{j}_{i}=\sum_{\boldsymbol{p} \sigma} \frac{\partial H_{\boldsymbol{p}+\boldsymbol{Q}_{i}}^{(i)}}{\partial \boldsymbol{p}} \mathcal{N}_{\boldsymbol{p}+\boldsymbol{Q}_{i}}^{(i)} .
$$

Since $\mathcal{N}_{\boldsymbol{p}+\boldsymbol{Q}_{\boldsymbol{i}}}^{(i)}$ differs from the equilibrium distribution $\mathcal{N}_{\boldsymbol{p} 0}^{(i)}$ only in the narrow region near the Fermi surface, Eq. (135) can be linearized and rewritten in the form, similar to Eq. (48), or, after some algebra, to Eq. (46). Then, using Eqs. (116) and (120) and noticing that

$$
\mathcal{N}_{\boldsymbol{p}+\boldsymbol{Q}_{i}}^{(i)}-\mathcal{N}_{-\boldsymbol{p}+\boldsymbol{Q}_{i}}^{(i)}=\mathcal{F}_{\boldsymbol{p}+\boldsymbol{Q}_{i}}^{(i)}-\mathcal{F}_{-\boldsymbol{p}+\boldsymbol{Q}_{i}}^{(i)}
$$


Eq. (135) can be finally presented as

$$
\boldsymbol{j}_{i}=\sum_{j} Y_{i j}\left[\boldsymbol{Q}_{j}+\frac{1}{n_{j}} \sum_{\boldsymbol{p} \sigma} \boldsymbol{p} \mathcal{F}_{\boldsymbol{p}+\boldsymbol{Q}_{j}}^{(j)}\right],
$$

where the matrix $Y_{i j}$ is given by Eq. (24). For a one-component Fermi-liquid Eq. (137) reduces to the well-known expression (see, e.g., Refs. [28, 54]),

$$
\boldsymbol{j}=\frac{n \boldsymbol{Q}}{m}+\sum_{\boldsymbol{p} \sigma} \frac{\boldsymbol{p}}{m} \mathcal{F}_{\boldsymbol{p}+\boldsymbol{Q}}
$$

To obtain this formula we employed Eq. (7) and the definitions (9) and (24).

It is important to emphasize that generally the mass current density $m_{i} \boldsymbol{j}_{i}$ of $i$-th particle species is not equal to the momentum density $\boldsymbol{P}_{i}$,

$$
\boldsymbol{P}_{i}=\sum_{\boldsymbol{p} \sigma}\left(\boldsymbol{p}+\boldsymbol{Q}_{i}\right) \mathcal{N}_{\boldsymbol{p}+\boldsymbol{Q}_{i}}^{(i)}=n_{i} \boldsymbol{Q}_{i}+\sum_{\boldsymbol{p} \sigma} \boldsymbol{p} \mathcal{F}_{\boldsymbol{p}+\boldsymbol{Q}_{i}}^{(i)}
$$

However, using Eq. (77) one can check that, due to Galilean invariance of the system, the following equality holds

$$
\sum_{i} m_{i} \boldsymbol{j}_{i}=\sum_{i} \boldsymbol{P}_{i}
$$

Now let us discuss the 'superfluid' equation. It has a natural form (see, e.g., Refs. 24, 28, 29, 54], where similar equations are written for a one-component liquid),

$$
\frac{\partial \boldsymbol{Q}_{i}}{\partial t}=-\nabla \breve{\mu}_{i}
$$

and coincides with the 'superfluid' equation (4.9) of Ref. [28]. (The authors of Ref. [28] used different notations. In particular, our quantity $\breve{\mu}$ is related to their invariant potential $\Phi$ by $\breve{\mu}=\mu-\Phi$, where $\mu$ is a constant from which the authors count energy.) Eq. (141) is also equivalent to the corresponding equation of the Khalatnikov's superfluid hydrodynamics [55],

$$
\frac{\partial \boldsymbol{V}_{\mathrm{s}}}{\partial t}=-\nabla\left(\mu_{\mathrm{Kh}}+\frac{V_{\mathrm{s}}^{2}}{2}\right) .
$$

To prove this, we notice that the Khalatnikov's chemical potential $\mu_{\mathrm{Kh}}$ (per particle mass $m$ ) is defined in a reference frame in which $V_{\mathrm{s}}=Q / m=0$, while our potential $\breve{\mu}$ is defined in the laboratory frame. They are related by an obvious formula, $\breve{\mu}=m\left(\mu_{\mathrm{Kh}}+V_{\mathrm{s}}^{2} / 2\right)$. Thus, Eqs. (141) and (142) are indeed equivalent.

Notice that Eq. (141) will be automatically satisfied if we express $\boldsymbol{Q}_{i}$ and $\breve{\mu}_{i}$ through the wave function phase $\varphi_{i}$ of the Cooper-pair condensate (see, e.g., Refs. 28, 47, 54]),

$$
\begin{aligned}
\boldsymbol{Q}_{i} & =\frac{1}{2} \nabla \varphi_{i}, \\
-\breve{\mu}_{i} & =\frac{1}{2} \frac{\partial \varphi_{i}}{\partial t} .
\end{aligned}
$$

One can verify that, in the linear theory considered in Sec. II, the phase $\varphi_{i}$ is related to the 'zero harmonic' of the function $O_{\boldsymbol{k} \sigma-}^{(i)}$ [see Eq. (38)] by the equation

$$
\varphi_{i}=\frac{i}{2 \Delta_{i}} \sum_{k} V_{0}^{(i)} \delta s_{\boldsymbol{k} \sigma-}^{(i)} .
$$

All other harmonics of $O_{\boldsymbol{k} \sigma-}^{(i)}$ are small and can be neglected in the quasiclassical limit [24]. 


\section{Inclusion of electromagnetic field and comparison with the previous works}

Eqs. (116), (117), (120)-(123), (125), (127), (128), (132), (133), (137), (139), (143), and (144) of Secs. IVA and IVB fully describe the two-component superfluid neutral Fermi-liquid in the limit of small $q$ and $\omega$. The generalization of these equations to the case of charged mixtures is straightforward (see, e.g., Refs. 28, 54]). Namely, they remain essentially the same if we redefine the quantities $\boldsymbol{Q}_{i}$ and $\breve{\mu}_{i}$ [see Eqs. (143) and (144)] through the gauge-invariant combinations,

$$
\begin{aligned}
\boldsymbol{Q}_{i} & =\frac{1}{2} \nabla \varphi_{i}-e_{i} \boldsymbol{A}, \\
-\breve{\mu}_{i} & =\frac{1}{2} \frac{\partial \varphi_{i}}{\partial t}+e_{i} V .
\end{aligned}
$$

Notice, however, that the 'superfluid' equation in the form (141) is no longer valid. As follows from Eqs. (146) and (147), it should be replaced by

$$
\frac{\partial \boldsymbol{Q}_{i}}{\partial t}=-\nabla \breve{\mu}_{i}+e_{i} \boldsymbol{E}
$$

where $\boldsymbol{E}=-\partial \boldsymbol{A} / \partial t-\nabla V$ is the self-consistent electric field, which can be found with the help of the Maxwell equations. The right-hand sides of Eqs. (146) and (147) are indeed gauge-invariant since the phase $\varphi_{i}$ transforms as $\varphi_{i} \rightarrow \varphi_{i}-2 e_{i} \phi$ under the gauge transformation (50) and (51) (see, e.g., Refs. 24, 47]).

The equations formulated above in Sec. IV reproduce various limiting cases that were studied in the literature. First of all, for a one-component Fermi-liquid our equations coincide with those obtained by Betbeder-Matibet and Nozieres [24] and by Aronov and Gurevich [54] (see also Refs. [28, 29]). Betbeder-Matibet and Nozieres worked in the linear approximation but assumed the most general form of the Landau interaction $f\left(\boldsymbol{k}, \boldsymbol{k}^{\prime}\right)$ and pairing potential $V\left(\boldsymbol{k}, \boldsymbol{k}^{\prime}\right)$. On the contrary, Aronov and Gurevich derived, from the first principles, the fully nonlinear system of equations, describing the superfluid Fermi-liquid in the quasiclassical regime. However, they completely neglected the Landau interaction $\left[f\left(\boldsymbol{k}, \boldsymbol{k}^{\prime}\right)=0\right]$ and took into account only the zero harmonic $V_{0}$ of the pairing interaction $\left(V_{l}=0\right.$ for $l>0)$.

Our equations for a two-component Fermi-liquid were compared only with the results of Sec. II since we did not find a discussion in the literature on transport properties of strongly interacting superfluid Fermi-mixtures. We checked that in the linear approximation our equations reproduce the quasiclassical limit of kinetic equations (33)-(36).

\section{SUMMARY}

This paper discusses transport properties of a mixture of two superfluid strongly interacting Fermi-liquids. A typical example of such mixture is the matter in the internal layers of neutron stars. To describe the mixture we use the Landau theory of Fermi-liquids generalized by Larkin and Migdal [42, 43] and by Leggett [23, 44] to take into account the effects of superfluidity.

Our results are summarized below.

(i) Working in the linear approximation, we formulate the system (33)-(36) of kinetic equations, describing the collisionless superfluid Fermi mixture in the self-consistent electromagnetic field. To derive these equations, we follow the approach of Betbeder-Matibet and Nozieres [24], who obtained the kinetic equation for a one-component superfluid Fermi-liquid. Generally, the system (33)-(36) is a straightforward generalization of the corresponding equations of Ref. [24]. However, there is one nontrivial difference concerning the form of interaction of Landau quasiparticles with the electromagnetic vector potential [see Eq. (22)]. For a one-component Fermi-liquid $\alpha_{i}$ in Eq. (22) is always equal to electric charge, $\alpha_{i}=e_{i}$, which is the consequence of the Galilean invariance of the system. On the contrary, for a multi-component mixture $\alpha_{i}$ is generally given by Eq. (23), while the Galilean invariance requires only that Eq. (7) must be satisfied.

(ii) Using the above kinetic equation, we determine the particle current density $\boldsymbol{j}_{i}$ of $i$-th particle species [see Eqs. (45), (46), or (48)] and show that it is given by the same expression as for a nonsuperfluid matter. For a one-component superfluid Fermi-liquid this was first shown by Leggett [44].

(iii) Assuming that the only two harmonics $f_{0}^{i j}$ and $f_{1}^{i j}$ of the Landau quasiparticle interaction $f^{i j}\left(\boldsymbol{k}, \boldsymbol{k}^{\prime}\right)$ are nonzero, we calculate the polarization functions $P_{00}^{(i)}, P_{1}^{(i)}$, and $P_{\mathrm{tr}}^{(i)}$ [see Eqs. (54), (70), and (71)], and compare them, in various limiting cases, with the results available in the literature (see, e.g., Refs. [9, 23, 38, 44]). We demonstrate, that the functions $P_{00,1, \operatorname{tr}}^{(i)}$ can be expressed through the Landau parameters and polarization functions $\Pi_{00,1, \operatorname{tr}}^{(i)}\left(p_{\mathrm{F} i}, v_{\mathrm{F} i}\right)$ of 
noninteracting Fermi-liquid, for which $f^{i j}\left(\boldsymbol{k}, \boldsymbol{k}^{\prime}\right)=0$. This result is valid for any smooth pairing potential $V^{(i)}\left(\boldsymbol{k}, \boldsymbol{k}^{\prime}\right)$ and for all wave vectors $\boldsymbol{q}$ and frequencies $\omega$ such that $q v_{\mathrm{F} i} \ll \mu_{j}$ and $\omega \ll \mu_{j}$.

(iv) We show that the transverse polarization function $P_{\mathrm{tr}}^{(i)}$ does not depend on the Landau quasiparticle interaction $f^{i j}\left(\boldsymbol{k}, \boldsymbol{k}^{\prime}\right)$ in the Pippard limit, when $q v_{\mathrm{F} j} \gg \Delta_{i}$ and $q v_{\mathrm{F} j} \gg \omega$. In this limit it is given by $P_{\mathrm{tr}}^{(i)}=\Pi_{\mathrm{tr}}^{(i)}\left(p_{\mathrm{F} i}, v_{\mathrm{F} i}\right)$. For a one-component Fermi-liquid and $\omega=0$ the same result was obtained previously by Leggett [44].

(v) Finally, we formulate a system of nonlinear equations describing the nonequilibrium superfluid mixture in the quasiclassical limit $\left(q v_{\mathrm{F} i} \ll \Delta_{j}\right.$ and $\left.\omega \ll \Delta_{j}\right)$. It consists of Eq. (125) for a nonequilibrium energy gap, scalar kinetic equation (132) for a Bogoliubov excitations, continuity equation (133), and of Eq. (148) for the superfluid velocity. In the linear approximation this system is completely equivalent to kinetic equations formulated in Sec. II and in Ref. [24]. Moreover, we verified that it reproduces the nonlinear equations derived from the first principles by Aronov and Gurevich [54] (see also Refs. [28, 29]). To simplify the problem, these authors neglected the Landau quasiparticle interaction and assumed that the pairing potential is a constant.

The results obtained in this paper can be useful in a variety of applications. For example, the polarization functions can be used to study low-frequency $\left(\omega \ll \mu_{i}\right)$ long-wavelength $\left(q v_{\mathrm{F} i} \ll \mu_{j}\right)$ collective modes in superfluid matter of neutron stars [see, e.g, recent papers [21, 22] for an example of such studies in the normal matter].

Also, the complex parts of the polarization functions $P_{00}^{(i)}$ and $P_{\mathrm{tr}}^{(i)}$ determine energy losses in the Cooper-pairing neutrino emission process. This process regulates thermal evolution of neutron stars [3, [56 [58] and is especially important in the crust of accreting neutron stars, exhibiting X-ray superbursts [59, 60].

Next, the kinetic equation derived in Sec. II can be used, with minor modification, to study the axial-vector response of a superfluid Fermi-liquid (see Sec. IIIB for more details). This problem is also important in application to the Cooper-pairing neutrino emission process [4, 6, 8, [9].

One needs the polarization functions in the Pippard limit since they describe the plasma screening of the interaction between charged particles (e.g., protons and electrons) in the collision integrals, determining the kinetic coefficients of neutron-star matter [33, 34].

Finally, the equations presented in Sec. IV, can be applied to study the response functions and collective modes in the equilibrium and in nonequilibrium matter (under the condition that $q v_{\mathrm{F} i} \ll \Delta_{j}$ and $\omega \ll \Delta_{j}$ ). Furthermore, after specifying the collision integral in Eq. (132) (see, e.g., Refs. [25 30, 54]), the equations of Sec. IV can be used to calculate the kinetic coefficients for a superfluid mixture, in particular, the thermal conductivity, shear and bulk viscosities. These coefficients are crucial for modeling of the dynamics of neutron stars [3, 61].

While doing this work we had in mind possible applications to neutron-star physics. However, the results obtained in this paper can be applied to any mixture of strongly interacting superfluid Fermi-liquids, for instance, to ultracold Fermi-Fermi mixtures, which have been realized recently [62, 63].

\section{Acknowledgments}

The author is very grateful to A. D. Kaminker and D. G. Yakovlev for reading the draft of the paper and valuable comments, and to L. B. Leinson for correspondence. This research was supported in part by RFBR (Grant 08-02-00837), and by the Federal Agency for Science and Innovations (Grant NSh 2600.2008.2). The author also acknowledges support from the Dynasty Foundation, the Mianowski Foundation, and from the RF Presidential Program (Grant MK-1326.2008.2).

\section{Appendix A}

Let us derive Eq. (23) for the coefficient $\alpha_{i}$. This coefficient enters the expression (22) for the matrix $\Lambda_{\boldsymbol{k} \sigma}^{(i)}$, describing the interaction of quasiparticles with the self-consistent electromagnetic field. Since this matrix is diagonal, it is sufficient to consider a mixture of strongly interacting normal Fermi-liquids. The kinetic equation (19) then reduces to

$$
\begin{aligned}
\omega \delta n_{\boldsymbol{k} \sigma}^{(i)} & =\left(\xi_{\boldsymbol{k}_{+}}^{(i)}-\xi_{\boldsymbol{k}_{-}}^{(i)}\right) \delta n_{\boldsymbol{k} \sigma}^{(i)}+\left(n_{\boldsymbol{k}_{-} 0}^{(i)}-n_{\boldsymbol{k}_{+} 0}^{(i)}\right) \sum_{\boldsymbol{k}^{\prime} \sigma^{\prime} j} f^{i j}\left(\boldsymbol{k}, \boldsymbol{k}^{\prime}\right) \delta n_{\boldsymbol{k}^{\prime} \sigma^{\prime}}^{(j)} \\
& +\left(n_{\boldsymbol{k}_{-} 0}^{(i)}-n_{\boldsymbol{k}_{+} 0}^{(i)}\right)\left(e_{i} V-\alpha_{i} \frac{\boldsymbol{k} \boldsymbol{A}}{m_{i}}\right)
\end{aligned}
$$

where $n_{\boldsymbol{k} 0}^{(i)}$ is given by Eq. (2) and we use the notation $\delta n_{\boldsymbol{k} \sigma}^{(i)} \equiv \delta n_{\boldsymbol{k} \sigma 11}^{(i)}=\left\langle a_{\boldsymbol{k}_{-} \sigma}^{(i) \dagger} a_{\boldsymbol{k}_{+} \sigma}^{(i)}\right\rangle$. 
The last term in the right-hand side of Eq. (149) appears due to the interaction of Landau quasiparticles with the self-consistent electromagnetic field. The corresponding interaction Hamiltonian has the form

$$
H_{\mathrm{em}}=\sum_{\boldsymbol{p} \sigma i}\left(e_{i} V-\alpha_{i} \frac{\boldsymbol{k} \boldsymbol{A}}{m_{i}}\right) a_{\boldsymbol{k}_{+} \sigma}^{(i) \dagger} a_{\boldsymbol{k}_{-} \sigma}^{(i)} .
$$

One can easily obtain this term using $H_{\mathrm{em}}$ and an equation of motion for the operator $a_{\boldsymbol{k}_{-} \sigma}^{(i) \dagger} a_{\boldsymbol{k}_{+} \sigma}^{(i)}$ (see, e.g., Ref. [24]).

In the limit of small $\boldsymbol{q}$, which is of our primary interest, Eq. (149) can be rewritten as

$$
\left(\omega-\boldsymbol{q} \boldsymbol{v}_{i}\right) \delta n_{\boldsymbol{k} \sigma}^{(i)}=-\frac{\partial n_{\boldsymbol{k} 0}^{(i)}}{\partial \boldsymbol{k}} \boldsymbol{q}\left[\sum_{\boldsymbol{k}^{\prime} \sigma^{\prime} j} f^{i j}\left(\boldsymbol{k}, \boldsymbol{k}^{\prime}\right) \delta n_{\boldsymbol{k}^{\prime} \sigma^{\prime}}^{(j)}+e_{i} V-\alpha_{i} \frac{\boldsymbol{k} \boldsymbol{A}}{m_{i}}\right] .
$$

The kinetic equation (151) [or (149)] is not obviously gauge-invariant. To make the gauge invariance explicit, one can notice that $k$ is actually a generalized momentum. It is related to the real momentum $\boldsymbol{p}$ of a quasiparticle $i$ by

$$
\boldsymbol{k}=\boldsymbol{p}+e_{i} A \text {. }
$$

For a very pedagogical discussion of the validity of this expression for Landau quasiparticles, see, e.g., Chapter 3 , $\S 6$ of Ref. [45].

Thus, we have two momentums, $\boldsymbol{k}$ and $\boldsymbol{p}$, and our next step will be to express the distribution function $\mathcal{N}_{\boldsymbol{p} \sigma}^{(i)} \equiv$ $n_{\boldsymbol{p} 0}^{(i)}+\delta \mathcal{N}_{\boldsymbol{p} \sigma}^{(i)}$ for quasiparticles with momentum $\boldsymbol{p}$ through the distribution function $n_{\boldsymbol{k} 0}^{(i)}+\delta n_{\boldsymbol{k} \sigma}^{(i)}$ for quasiparticles with momentum $\boldsymbol{k}$. Because of the one-to-one correspondence between $\boldsymbol{k}$ and $\boldsymbol{p}$, one has

$$
\mathcal{N}_{\boldsymbol{p} \sigma}^{(i)}=n_{\boldsymbol{k} 0}^{(i)}+\delta n_{\boldsymbol{k} \sigma}^{(i)} .
$$

In view of Eq. (152), in the linear approximation,

$$
n_{\boldsymbol{k} 0}^{(i)}=n_{\boldsymbol{p}+e_{i} \boldsymbol{A} 0}^{(i)} \approx n_{\boldsymbol{p} 0}^{(i)}+\frac{\partial n_{\boldsymbol{p} 0}^{(i)}}{\partial \boldsymbol{p}} e_{i} \boldsymbol{A}
$$

It follows then from Eq. (153)

$$
\delta n_{\boldsymbol{k} \sigma}^{(i)}=\delta \mathcal{N}_{\boldsymbol{p} \sigma}^{(i)}-\frac{\partial n_{\boldsymbol{p} 0}^{(i)}}{\partial \boldsymbol{p}} e_{i} \boldsymbol{A}
$$

Substituting Eq. (155) into the kinetic equation (151) and demanding that the terms which are noninvariant under gauge transformations vanish, one obtains the condition

$$
\boldsymbol{q} \boldsymbol{v}_{i}\left[\frac{\partial n_{\boldsymbol{p} 0}^{(i)}}{\partial \boldsymbol{p}} e_{i} \boldsymbol{A}\right]=\frac{\partial n_{\boldsymbol{p} 0}^{(i)}}{\partial \boldsymbol{p}} \boldsymbol{q}\left[\sum_{\boldsymbol{p}^{\prime} \sigma^{\prime} j} f^{i j}\left(\boldsymbol{p}, \boldsymbol{p}^{\prime}\right) \frac{\partial n_{\boldsymbol{p}^{\prime} 0}^{(j)}}{\partial \boldsymbol{p}^{\prime}} e_{j} \boldsymbol{A}+\alpha_{i} \frac{\boldsymbol{p} \boldsymbol{A}}{m_{i}}\right]
$$

or, after performing an integration, the expression (23) for $\alpha_{i}$. For a one-component Fermi-liquid Eq. (23) simplifies with the help of Eq. (7) and one gets $\alpha_{i}=e_{i}$. [45]),

Using Eq. (156), the kinetic equation (151) can be recasted in the well-known gauge-invariant form (see, e.g., Ref.

$$
\left(\omega-\boldsymbol{q} \boldsymbol{v}_{i}\right) \delta n_{\boldsymbol{p} \sigma}^{(i)}=-\frac{\partial n_{\boldsymbol{p} 0}^{(i)}}{\partial \boldsymbol{p}}\left[\boldsymbol{q} \sum_{\boldsymbol{p}^{\prime} \sigma^{\prime} j} f^{i j}\left(\boldsymbol{p}, \boldsymbol{p}^{\prime}\right) \delta n_{\boldsymbol{p}^{\prime} \sigma^{\prime}}^{(j)}+i e_{i} \boldsymbol{E}\right],
$$

where $\boldsymbol{E}=i(\omega \boldsymbol{A}-\boldsymbol{q} V)$ is the electric field.

\section{Appendix B}

Let us derive Eq. (64) directly for a one-component Fermi-liquid. In this case one has to put $f_{0}^{i j}=0$ and $f_{1}^{i j}=0$ for $i \neq j$ in all equations. In what follows we suppress the particle species indices to simplify notations. We start with Eq. (62) which can be rewritten as

$$
A_{\boldsymbol{k} \sigma}=2 e \frac{\boldsymbol{k} \boldsymbol{A}}{m}-\frac{\boldsymbol{k}}{k_{\mathrm{F}}^{2}} f_{1} \boldsymbol{I} \equiv 2 e \frac{\boldsymbol{k} \boldsymbol{A}_{\mathrm{eff}}}{m^{*}},
$$


where we used the fact that $\alpha=e$ for a one-component Fermi-liquid (see Appendix A) and defined

$$
\boldsymbol{I} \equiv \sum_{\boldsymbol{k}^{\prime} \sigma^{\prime}} \boldsymbol{k}^{\prime} \delta n_{\boldsymbol{k}^{\prime} \sigma^{\prime}+}
$$

The quantity $\boldsymbol{I}$ is not gauge-invariant. Our aim will be to express $\boldsymbol{I}$ through the vector potential $\boldsymbol{A}$ and the gaugeinvariant particle current density $\boldsymbol{j}$, which is given by Eq. (46). In view of the definition (159), Eq. (46) can be rewritten as

$$
\boldsymbol{j}=\frac{1}{2}\left[\frac{\boldsymbol{I}}{m^{*}}-\sum_{\boldsymbol{k} \sigma} \frac{\partial \mathcal{N}_{\boldsymbol{k} 0}}{\partial k} f_{1} \frac{k_{\alpha} k_{\beta}}{k_{\mathrm{F}}^{3}} I_{\beta}\right]-e \frac{n}{m} \boldsymbol{A} .
$$

Here $\alpha$ and $\beta$ are the space indices. The integral in Eq. (160) can be easily taken since the function $\partial \mathcal{N}_{\boldsymbol{k}} 0 / \partial k$ has a sharp maximum near the Fermi-surface. As a result, we obtain

$$
\boldsymbol{j}=\frac{1}{2}\left[\frac{1}{m^{*}}+\frac{k_{\mathrm{F}} f_{1}}{3 \pi^{2}}\right] \boldsymbol{I}-e \frac{n}{m} \boldsymbol{A} .
$$

As follows from Eq. (7) for the effective mass, the expression in square brackets equals $1 / m$. Thus, one finds

$$
\boldsymbol{j}=\frac{1}{2 m} \boldsymbol{I}-e \frac{n}{m} \boldsymbol{A}
$$

or

$$
\boldsymbol{I}=2 m\left(\boldsymbol{j}+e \frac{n}{m} \boldsymbol{A}\right)
$$

Substituting this formula into Eq. (158), one gets

$$
A_{\boldsymbol{k} \sigma}=2 e\left(\frac{1}{m}-\frac{n f_{1}}{k_{\mathrm{F}}^{2}}\right) \boldsymbol{k} \boldsymbol{A}-\frac{2 m f_{1}}{k_{\mathrm{F}}^{2}} \boldsymbol{k} \boldsymbol{j} \equiv 2 e \frac{\boldsymbol{k} \boldsymbol{A}_{\mathrm{eff}}}{m^{*}} .
$$

Again, using Eq. (77), it follows that the expression in brackets equals $1 / m^{*}$. Eq. (164) can then be rewritten as

$$
A_{\boldsymbol{k} \sigma}=\frac{2 e}{m^{*}} \boldsymbol{k} \boldsymbol{A}-\frac{2 m f_{1}}{k_{\mathrm{F}}^{2}} \boldsymbol{k} \boldsymbol{j} \equiv 2 e \frac{\boldsymbol{k} \boldsymbol{A}_{\mathrm{eff}}}{m^{*}} .
$$

That is

$$
\boldsymbol{A}_{\mathrm{eff}}=\boldsymbol{A}-\frac{1}{e} \frac{m m^{*}}{k_{\mathrm{F}}^{2}} f_{1} \boldsymbol{j}
$$

This equation coincides with Eq. (64) if the latter is written for a one-component Fermi-liquid.

[1] D. G. Yakovlev, K. P. Levenfish, and Yu. A. Shibanov, Physics-Uspekhi 42, 737 (1999).

[2] U. Lombardo and H.-J. Schulze, Lect. Notes Phys. 578, 30 (2001).

[3] D. G. Yakovlev and C. J. Pethick, Ann. Rev. Astron. Astrophys. 42, 169 (2004).

[4] J. Kundu and S. Reddy, Phys. Rev. C70, 055803 (2004).

[5] A. Sedrakian, H. Müther, P. Schuck, Phys. Rev. C76, 055805 (2007).

[6] E. E. Kolomeitsev and D. N. Voskresensky, Phys. Rev. C77, 065808 (2008).

[7] L. B. Leinson, Phys. Rev. C78, 015502 (2008).

[8] A. W. Steiner and S. Reddy, Phys. Rev. C79, 015802 (2009).

[9] L. B. Leinson, Phys. Rev. C79, 045502 (2009).

[10] L. B. Leinson, A. Pérez, Nucl. Phys. B597, 279 (2001).

[11] P. Haensel, Nucl. Phys. A301, 53 (1978).

[12] F. Matera and V. Yu. Denisov, Phys. Rev. C49, 2816 (1994).

[13] G. Fabbri and F. Matera, Phys. Rev. C54, 2031 (1996).

[14] A. Burrows and R. F. Sawyer, Phys. Rev. C58, 554 (1998).

[15] S. Reddy, M. Prakash, J. M. Lattimer, and J. A. Pons, Phys. Rev. C59, 2888 (1999). 
[16] V. Greco, M. Colonna, M. di Toro, and F. Matera, Phys. Rev. C67, 015203 (2003).

[17] C. Shen, U. Lombardo, N. van Giai, and W. Zuo, Phys. Rev. C68, 055802 (2003).

[18] C. Providência, L. Brito, A. M. S. Santos, D. P. Menezes, and S. S. Avancini, Phys. Rev. C74, 045802 (2006).

[19] G. I. Lykasov, C. J. Pethick, and A. Schwenk, Phys. Rev. C78, 045803 (2008).

[20] C. Ducoin, J. Margueron, and Ph. Chomaz, Nucl. Phys. A809, 30 (2008).

[21] M. Baldo and C. Ducoin, Physics of Atomic Nuclei, 72, 1188 (2009).

[22] M. Baldo and C. Ducoin, Phys. Rev. C79, 035801 (2009).

[23] A. J. Leggett, Phys. Rev. 147, 119 (1966).

[24] O. Betbeder-Matibet and P. Nozières, Ann. Phys. 51, 392 (1969).

[25] P. Wölfle, J. Low Temp. Phys. 22, 157 (1976).

[26] M. J. Stephen, Phys. Rev. 139, 197 (1965).

[27] B. T. Geilikman and V. Z. Kresin, Usp. Fiz. Nauk 99, 51 (1969) [Sov. Phys. Usp. 12, 620 (1970)].

[28] A. G. Aronov, Y. M. Galperin, V. L. Gurevich, and V. I. Kozub, Adv. Phys. 30, 539 (1981).

[29] A. G. Aronov, Y. M. Galperin, V. L. Gurevich, and V. I. Kozub, Nonequilibrium properties of Superconductors - Transport Equation Approach, edited by D. N. Langenberger and A. I. Larkin (Elsevier, New York, 1986), p. 325.

[30] N. B. Kopnin, Theory of Non-Equilibrium Superconductivity (Oxford University Press, 2001)

[31] O. Y. Gnedin and D. G. Yakovlev, Nucl. Phys. A582, 697 (1995).

[32] D. A. Baiko, P. Haensel, and D. G. Yakovlev, Astron. Astrophys. 374, 151 (2001).

[33] P. S. Shternin and D. G. Yakovlev, Phys. Rev. D75, 103004 (2007).

[34] P. S. Shternin and D. G. Yakovlev, Phys. Rev. D78, 063006 (2008).

[35] D. N. Aguilera, V. Cirigliano, J. A. Pons, S. Reddy, and R. Sharma, Phys. Rev. Lett. 102, 091101 (2009).

[36] A. F. Andreev and E. P. Bashkin, Zh. Eksp. Teor. Fiz. 69, 319 (1975) [Sov. Phys. JETP 42, 164 (1976)].

[37] M. Borumand, R. Joynt, and W. Kluźniak, Phys. Rev. C54, 2745 (1996).

[38] M. E. Gusakov and P. Haensel, Nucl. Phys. A761, 333 (2005).

[39] N. Chamel and P. Haensel, Phys. Rev. C73, 045802 (2006).

[40] M. E. Gusakov, Phys. Rev. D76, 083001 (2007).

[41] N. Chamel and P. Haensel, Liv. Rev. Rel., 11, 1 (2008).

[42] A. I. Larkin and A. B. Migdal, Zh. Eksp. Teor. Fiz. 441703 (1963) [Sov. Phys. JETP 171146 (1963)].

[43] A. I. Larkin, Zh. Eksperim. i Teor. Fiz. 46, 2188 (1964) [Soviet Phys. JETP 19, 1478 (1964)].

[44] A. J. Leggett, Phys. Rev. A140 (1965) 1869.

[45] D. Pines, P. Nozières, Theory of Quantum Liquids (Benjamin, New York, 1966).

[46] G. Baym and C. J. Pethick, Landau Fermi-Liquid Theory: Concepts and Applications (Wiley, New York, 1991).

[47] E. M. Lifshitz and L. P. Pitaevskii, Statistical Physics, Part 2 (Pergamon, Oxford, 1980).

[48] M. E. Gusakov, E. M. Kantor, and P. Haensel, Phys. Rev. C79, 055806 (2009).

[49] M. E. Gusakov, E. M. Kantor, and P. Haensel, Phys. Rev. C80, 015803 (2009).

[50] Y. Nambu, Phys. Rev. 117, 648 (1960).

[51] E. M. Lifshitz and L. P. Pitaevskii, Physical Kinetics (Pergamon, Oxford, 1981).

[52] P. I. Arseev, S. O. Loiko, and N. K. Fedorov, Physics Uspekhi 49, 1 (2006).

[53] L. B. Leinson, private communication (2010).

[54] A. G. Aronov and V. L. Gurevich, Solid State Physics 16, 2656 (1974).

[55] I. M. Khalatnikov, An Introduction to the Theory of Superfluidity (Addison-Wesley, New York, 1989).

[56] M. E. Gusakov, A. D. Kaminker, D. G. Yakovlev, and O. Y. Gnedin, Astron. Astrophys. 423, 1063 (2004).

[57] D. Page, J. M. Lattimer, M. Prakash, and A. W. Steiner, Astrophys. J. Suppl. 155, 623 (2004).

[58] D. Page, J. M. Lattimer, M. Prakash, and A. W. Steiner, arXiv:0906.1621 (2009).

[59] E. F. Brown and A. Cumming, Astrophys. J., 698, 1020 (2009).

[60] L. Keek, J. J. M. in't Zand, E. Kuulkers, A. Cumming, E. F. Brown, and M. Suzuki, Astron. Astrophys. 479, 177 (2008).

[61] N. Andersson, Astrophys. Space Sci. 308, 395 (2007).

[62] M. Taglieber, A.-C. Voigt, T. Aoki, T. W. Hänsch, and K. Dieckmann, Phys. Rev. Lett. 100, 010401 (2008).

[63] E. Wille et al., Phys. Rev. Lett. 100, 053201 (2008). 